\title{
A Locked Mode Indicator for Disruption Prediction on JET and ASDEX Upgrade
}

\author{
G. Sias ${ }^{1}$, B. Cannas ${ }^{1}$, A. Fanni ${ }^{1}$, A Murari ${ }^{2}$, A. Pau ${ }^{1}$, the ASDEX Upgrade Team ${ }^{3 *}$, the EUROfusion \\ MST1 Team ${ }^{* *}$ and the JET Contributors *** \\ EUROfusion Consortium, JET, Culham Science Centre, Abingdon, OX14 3DB, UK \\ ${ }^{1}$ Electrical and Electronic Engineering Dept.-University of Cagliari, Piazza D’Armi, 09123, Cagliari, Italy. \\ ${ }^{2}$ Consorzio RFX (CNR, ENEA, INFN, Universita' di Padova, Acciaierie Venete SpA), Corso Stati Uniti 4, 35127 \\ Padova, Italy. \\ ${ }^{3}$ Max-Planck-Institüt fur Plasmaphysik, EURATOM Association, Garching - Germany \\ * See the author list "A. Kallenbach et al., Nucl. Fusion 57 102015" \\ ** See the author list "H. Meyer et al 2017 Nucl. Fusion 57 102014" \\ **** See the author list of "X. Litaudon et al 2017 Nucl. Fusion 57102001
}

\begin{abstract}
The aim of this paper is to present a signal processing algorithm that, applied to the raw Locked Mode signal, allows us to obtain a disruption indicator in principle exploitable on different tokamaks. A common definition of such an indicator for different machines would facilitate the development of portable systems for disruption prediction, which is becoming of increasingly importance for the next tokamak generations. Moreover, the indicator allows us to overcome some intrinsic problems in the diagnostic system such as drift and offset. The behavior of the proposed indicator as disruption predictor, based on crossing optimized thresholds of the signal amplitude, has been analyzed using data of both JET and ASDEX Upgrade experiments. A thorough analysis of the disruption prediction performance shows how the indicator is able to recover some missed and tardy detections of the raw signal. Moreover, it intervenes and corrects premature or even wrong alarms due to, e.g., drifts and/or offsets.
\end{abstract}

\section{Introduction}

Rotating magneto-hydro-dynamic (MHD) modes in tokamaks are often observed to slow down as their amplitude grows up. When a critical threshold is reached, they stop rotating, or, as it is commonly said, they lock in a certain toroidal and poloidal position leading very often to disruption or, in any case, to a degradation of confinement. The slowing down of the mode propagation velocity can be detected directly measuring the voltage induced by the oscillating 
field perturbation in tangential field pick up coils or Mirnov coils. Before the mode locking, the measured signal exhibits a growing amplitude accompanied by a reduction in frequency until the oscillation disappears, that is when the mode locks. When the mode is locking the amplitude of its radial component can be evaluated through the measure of the voltage induced in the saddle coils. Such amplitude underlies the analysis developed in this work. In a tokamak device, the mode locking is the most frequent precursor of disruptions, even if, in JET-ILW campaigns, an increased rate of disruptions driven by core radiation peaking or impurities has been observed [1], [2]. Usually, mode locking appears at a later stage in the chain of events characterizing the disruptive process. Its process can be caused by intrinsic error fields or by the deceleration of rotating precursor modes [1]. The analysis of these instabilities can be exploited to develop disruption predictors provided that they have an amplitude large enough to be detected before the thermal quench.

As reported in [1], [3], [4], most disruptions on JET present precursor locked modes that can be due to either error fields or initially rotating modes. However, for a non-negligible number of them, they manifest too late to intervene. On ASDEX Upgrade (AUG), it is more common to see modes that lock prior to the thermal quench, but also modes that are still rotating at the time of the thermal quench. Such difference can be justified by the lower intrinsic error fields in ASDEX Upgrade [5] compared to JET. Also on AUG, in several percent of cases, the instability manifests itself too close to the disruption time to allow any intervention. An analysis of the locking of the mode before the disruption in the databases of JET and AUG is reported in Section 4.

On JET and AUG, protections against locked mode disruptions already exist, which use the amplitude of the locked mode signal as a threshold. Such protection systems are machine dependent; at AUG the raw signal, available in real time for disruption mitigation purposes, provides a measurement in Volt, whereas at JET such signal is in Tesla.

However, the development of portable systems for disruption prediction is becoming of increasingly importance for the next tokamak generations. Referring to JET and AUG, in this paper, a common definition of a locked mode indicator is proposed and the viability to identify a common threshold value is investigated. To this purpose, referring to the JET and AUG experiments, a multi-machine analysis has been performed. In particular, two databases have been built containing about six hundreds of disrupted and non-disrupted discharges in JET with ITER-Like Wall (ILW) from 2012 to 2014 and roughly the same number of shots at AUG from 2009 to 2015. The AUG raw signal has been calibrated in Tesla to be compared with that of JET. 
Moreover, in order to handle dimensionless quantities both the signals at JET and AUG have been normalized as suggested in [3].

Furthermore, in both machines, the raw signals sometimes show offsets and/or drifts, due to no compensation of the poloidal fluxes generated by the plasma current and the current driving the $\mathrm{OH}$ coils. Moreover, active coils for error field correction could introduce off-set that have to be carefully handled. Hence, to avoid the effect of spurious trips in this signal, the trigger level used to request the pulse termination is set to a rather high value. This procedure has the clear drawback of delaying the request to safely shut down the plasma even when, by monitoring the signal itself, the presence of a locked mode could have been detected earlier.

Usually, the roots of drift and off-set are well known, and the correction could be trivial. However, the analysis of their causes could be time consuming and its results do not enhance the disruption prediction capability of the available signal. Hence, removing the drift and the off-set from the point of view of the real time data processing, without getting into the diagnostic limits and machine settings, is sufficient. In this paper, an algorithm for drift and off-set removal has been implemented and applied to the normalized locked mode signal. The algorithm does not require any preliminary analysis, and it can be applied also to those shots not affected by drift and off-set preserving the signal information one is interested in.

Finally, as proposed in the literature [6] [7], the resulting locked mode signal has been processed to exploit the content information of both the time and frequency domains resulting in an indicator fitting both devices.

In fact, some algorithms, mostly based on the frequency content of the raw locked mode signal, have been tested at JET for minimizing the effect of these issues so as to provide an earlier alarm than the current locked mode protection system [6]. These algorithms demonstrated to perform better than those that trigger an alarm only when the locked mode amplitude exceeds a prefixed threshold. Also in [7] time-frequency analysis was used to detect anomalies in the locked mode signal that trigger the disruption alarms on JET. Such predictor required a limited number of past experiments to set the alarm threshold.

In the present paper, the validity of the proposed indicator has been assessed by using it as disruption predictor.

Finally, a deep analysis of the prediction errors has been performed to understand the intrinsic limits of the use of only the locked mode signal as a disruption predictor. 
The remainder of this paper is organized as follows. Section 2 reports details of the locked mode diagnostics at JET and AUG. The rationale of the locked mode indicator is presented in Section 3. The statistical analysis of the adopted databases is presented in Section 4. Results of the locked mode indicator as disruption predictor, and a reasoned analysis of the obtained results are discussed in Section 5. In Section 6 conclusions are drawn.

\section{Locked Mode diagnostics}

On JET, the Locked Mode amplitude is measured by a set of $2 \times 4$ saddle flux loops, located at radial positions, above and below the middle plane, and mounted on the outside of the vacuum vessel at the low-field side (LFS) of the plasma. They are positioned at a $90^{\circ}$ angle to each other. The locked mode amplitude, measure of the predominant odd $n=1$ mode, comes from several elaborations of these flux loops signals, such as integration and the appropriate compensation of the poloidal fluxes generated by the plasma current and the current driving the $\mathrm{OH}$ coils. It is the effective amplitude of the radial component of the mode, being the two measurements the sine $\left(B r_{M H D F}\right)$ and cosine $\left(B r_{M H D G}\right)$ components of the mode [4]. The resulting locked mode amplitude signal is calculated as indicated in equation (1), it is in Tesla and will be indicated in the following with $B_{M L}$.

$$
B_{L M}=\sqrt{\left(B r_{M H D G}\right)^{2}+\left(B r_{M H D F}\right)^{2}}
$$

Regarding AUG, the signals for the present study come from a diagnostic constituted by two magnetic saddle coils mounted oppositely at the high-field side of the vacuum vessel. Each coil consists of one loop covering approximately $180^{\circ}$ of the vacuum vessel wall, measuring difference between the time derivative of the radial magnetic fields $B^{e}{ }_{r}$ and $B^{w}{ }_{r}$ on the coils located at the east and west side of the torus respectively. The resulting signal, which is not calibrated, provides a measurement in Volt (2):

$$
\frac{d B_{r}^{e w}}{d t}=\frac{d B_{r}^{e}}{d t}-\frac{d B_{r}^{w}}{d t}
$$

and it contains again information on the locked mode amplitude. In the considered shot range, the routinely used disruption trigger made reference to this diagnostic. Since, the paper aims to improve the prediction capability of the raw signal actually used as predictor, such diagnostic has been chosen as reference. In the following this raw signal will be labelled LM-raw. 
It is worth noting that, as the considered measure in AUG is based only on a couple of opposite saddle coils, the spatial resolution could be not enough if the phase of the locking position is not favorable with respect to the area covered by the loops. This problem has been recently solved on AUG by implementing a new diagnostic that is constituted by a set of 4 saddle coils on the low field side (LFS) [5].

Another important difference between JET and AUG locked mode amplitude diagnostics is that on AUG the signal is uniformly sampled at 2 MSamples/s whereas at JET the sampling time can vary during a discharge from several tens of Samples/s to some kSamples/s.

Note that, the LM diagnostic signal used for JET [8] is not the reference signal for real time applications, but the analysis developed in the following can be easily applied to the raw signals coming from real time diagnostics.

\section{Locked Mode Indicator}

Generally speaking, an indicator can synthesize more signals in order to describe a complex phenomenology, or can be based on a single signal when the signal itself is intrinsically representative of a disruptive behaviour, as for example, in the case of the locked mode. In most circumstances, in order to maximize the information content in the signal, it can be required to remove noise or unwanted spikes, or simply to extract the trend of the signal filtering transient phenomena. In this paper, the Locked Mode amplitude raw signals have been processed in the time and frequency domains in order to synthesize an indicator (called $L M$-ind) suitable for both JET and AUG tokamaks.

Firstly, the difference in the unit of measurement between JET and AUG raw signals requires suitable corrections in order to scale the MHD perturbation amplitude threshold to trigger a disruption predictor for the next tokamak generation machines, as proposed in [3]. In the present work, the absolute value of the AUG Locked Mode raw signal has been calibrated in order to convert it in Tesla, as reported in (3):

$$
B_{M L}(\mathrm{t})=\frac{1}{A} \int_{t-\Delta t}^{t} L M-r a w d t
$$

where $A=1.426389 m^{2}$ is the coil surface, and $\Delta t=10 m s$ is interval of integration.

Then, in order to allow a possible comparison among the two machines, a suitable raw signal normalization has been implemented. Even if the amplitude of the LM signal scales with several 
quantities, in this work, as suggested in [3], the normalization has been done with respect to the averaged edge poloidal field between HFS (at $R-a$ ), and LFS (at $R+a$ ) at the middle plane, $B_{\theta}(0, a)$, in [T], where $R$ is the major radius, and $a$ is the minor radius, measured at each sampling time. Such normalization provides a machine independent dimensionless parameter $B_{M L} / B_{\theta}(0, a)$. Note that, in this paper, $B_{M L}$ indicates both the Locked Mode raw signal in $[\mathrm{T}]$ at JET, and the Locked Mode raw signal calibrated in $[\mathrm{T}]$ at AUG.

$L M$-ind is obtained by multiplying the following three scalar features resulting from the processing of $B_{M L} / B_{\theta}(0, a)$ both in time and frequency domains. At each time instant $t_{k}$ :

- the first feature is the normalized raw amplitude, in [T], after detrending and off-set removal:

$$
f_{1}\left(t_{k}\right)=\left|B_{M L} / B_{\theta}(0, a)\left(t_{k}\right)-\bar{M}_{k}\right|
$$

where $\bar{M}_{k}$ is the mean value of the signal evaluated in the previous $3.2 \mathrm{~ms}$.

- the Fast Fourier Transform (FFT) of $B_{M L} / B_{\theta}(0, a)$ in the previous $51.2 m s$ is performed, and the standard deviation of the resulting signal frequency spectrum (after discarding the DC one) is taken into account as second feature:

$$
f_{2}\left(\mathrm{t}_{k}\right)=\sqrt{\frac{\sum_{m=1}^{P / 2}\left(\mathrm{~F}_{m}-\bar{F}\right)}{P / 2}}
$$

where $F_{m}$ is the amplitude of the $m^{\text {th }}$ component of the frequency spectrum, and $\bar{F}$ is the mean value of the $P / 2$ considered components. $P=512$ is the number of samples in the considered time window, being the sampling frequency equal to $10 \mathrm{kHz}$.

- the sum of amplitude of the frequency spectrum components, weighted with respect to the inverse of their squared frequencies, is evaluated as third feature:

$$
f_{3}\left(t_{k}\right)=\sum_{m=1}^{P / 2} \frac{1}{f^{2}} \mathrm{~F}_{m}
$$

Then, in order to develop a locked mode indicators exploitable in both machines, both $B_{M L} / B_{\theta}(0, a)$ and $L M$-ind signals have been re-sampled at $1 \mathrm{kSamples} / \mathrm{s}$ by means of a linear interpolation. Note that, the variable sampling frequency in JET diagnostic could reverberate on the results of a locked mode indicator if it is based also on the frequency content. This aspect has been carefully considered in the definition of the proposed Locked Mode indicator. 
Figure 1 reports the result of the algorithm for a JET disrupted pulse. In particular, Figure 1-a reports the raw LM signal $\left(B_{M L}\right)$, Figure 1-b shows the related LM indicator (LM-ind), whereas the subplots c) and d) report a zoom of the subplots a) and b) respectively, in the time window $63 \div 65.26 s$. Figure 2 reports the results of the algorithm applied on an AUG disruptive pulse, showing similar behaviour. For both figures, comparing plots a) and b) it can be observed as the indicator highlights, more than the raw signal, the mode locking phase with respect the behaviour of the rest of the shot. Whereas, comparing the two zooms, reported in figure c) and d), for JET it can be noted that the indicator is not affected by the variable sampling frequency of the diagnostic; for AUG, it can be noted that the indicator highlights the beginning of the mode locking phase before the raw signal.

84166
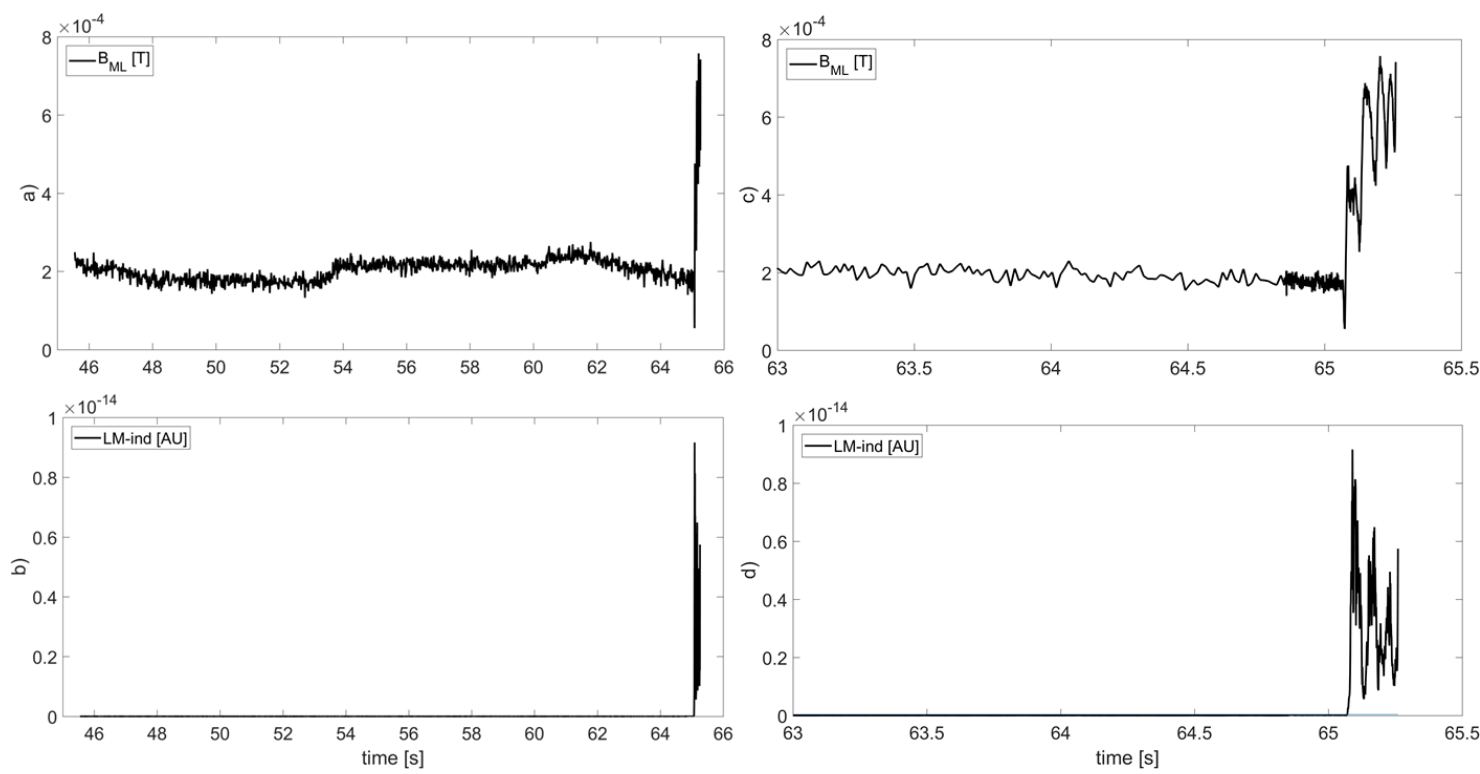

Figure 1. An example of JET disrupted pulse: (a) Locked Mode raw signal $B_{M L}$ in [T], (b) the LM indicator in arbitrary units [AU]. The subplots c) and d) report a zoom (time window $63 \div 65.26 s$ ) of the subplots a) and b) respectively. 

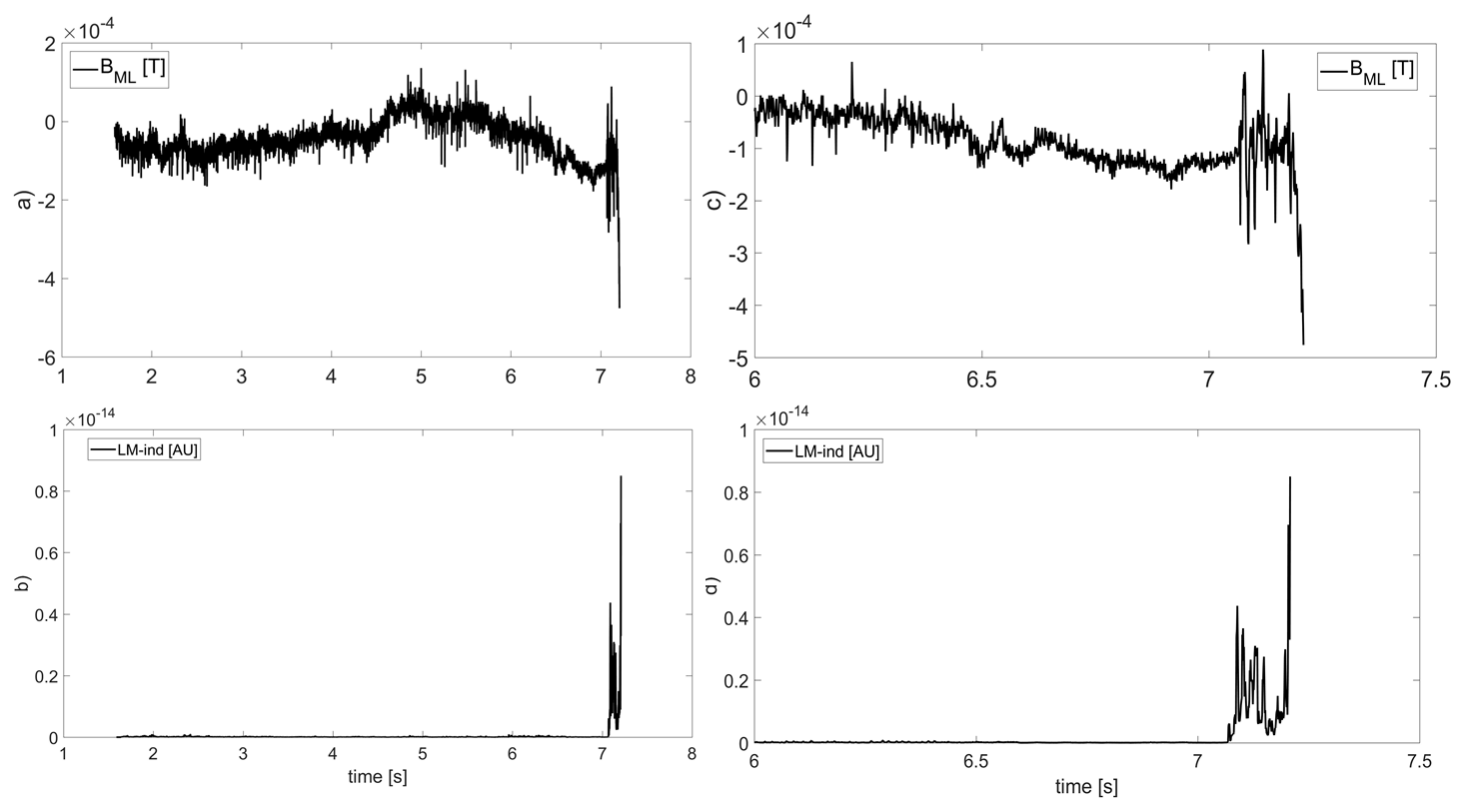

Figure 2. An example of AUG disrupted pulse: (a) Locked Mode raw signal $B_{M L}$ in [T], (b) the LM indicator in arbitrary units [AU]. The subplots c) and d) report a zoom (time window $6 \div 7.21 s$ ) of the subplots a) and b) respectively.

\section{Databases}

In order to statistically assess the effectiveness and robustness of the proposed indicator, it has been tested as a disruption predictor with both JET and AUG data. For the sake of comparison, the performance of the prediction systems, obtained by optimizing a threshold on the actual locked mode trigger, i.e., the raw signal normalized with respect to the plasma current $I_{p l a}$ on JET $\left(B_{L M} / I_{\text {pla }}\right)$ in $[\mathrm{T} / \mathrm{MA}]$, and the raw signal (LM-raw) in $[\mathrm{V}]$ at $\mathrm{AUG}$, have been computed. Moreover, also the dimensionless parameters $B_{L M} / B_{\theta}(0, a)$ in both the machines have been considered as reference.

Data for this study was selected from the ITER Like Wall (ILW) experimental campaigns performed at JET from 2012 to 2014 and at AUG from 2009 to 2015. Table 1 reports the composition of the JET database, whereas Table 2 refers to AUG data. 
Table 1. JET database

\begin{tabular}{lccc}
\hline \multicolumn{1}{c}{ Set } & $\begin{array}{c}\text { ILW experimental } \\
\text { campaigns }\end{array}$ & DISR & NO-DISR \\
& $2012-2012$ & & \\
\hline Training & $2012-2014$ & 54 & 84 \\
Test & & 210 & 251 \\
\hline
\end{tabular}

Both tables report the experimental campaigns, and the number of the disruptive (DISR) and regularly terminated (NO-DISR) shots selected for the training and the test sets.

The training set of JET was extracted from the experimental campaigns performed with the ILW and it contains only flat top disruptions excluding those terminated by massive gas injection. The 54 disruptions were manually and automatically classified [1], [9]. In order to test both the ageing and the generalization capability of the LM-ind as disruption predictor, a larger test set has been built, which covers a wide range of campaigns from 2012 to 2014 and contains not only flat-top disruptions. All disruptions occurred in the chosen experimental campaigns have been included with the exception of disruptions following vertical displacement events (VDEs) and those intentionally performed for the characterization of massive gas injection (MGI). The database contains the disruption times $\left(\mathrm{t}_{\mathrm{D}}\right)$ calculated by using an automatic procedure that is part of a tool (DIS_tool) available for both JET and AUG [10].

It has to be highlighted that a very important aspect in disruption prediction issue is to design a system able, not only to correctly predict disruptions, but also to minimize false alarms on regularly terminated discharges. To this purpose, 84 non-disrupted pulses have been used to train the system, belonging to the same range of the training disruptive pulses, and other 251 nondisrupted pulses, available from the experimental campaigns from 2012 to 2014, have been selected for the test set.

The same criteria employed at JET have been used to select non-disrupted and disrupted discharges on AUG. The training set contains only flat top disruptions whereas the test set contains also no-flat top disruptions and it extends over a larger time period (see Table 2). 
Table 2. AUG database

\begin{tabular}{lccc}
\hline Set & Experimental campaign & DISR & NO-DISR \\
\hline Training & $2009-2013$ & 60 & 72 \\
Test & $2009-2015$ & 222 & 254 \\
\hline
\end{tabular}

\section{Analysis of the Locked Mode signal}

A manual analysis has been performed to verify the possible presence of the locked mode for each disruptive shot in the database and the locked mode time (LM time) has been recorded. At JET it is assumed as the time instant where the absolute value of the signal starts to rise, regardless of its amplitude, whereas at AUG, the LM time is the time instant where the frequency of oscillation of the raw signal decreases and its absolute value starts to rise. Figure 3 and Figure 4 report examples of the locked mode raw signal of a JET and an AUG disrupted pulse respectively. The LM time has been manually set corresponding to the vertical dashed line. The red vertical line refers to the disruption time tD automatically calculated by DIS_tool [10].

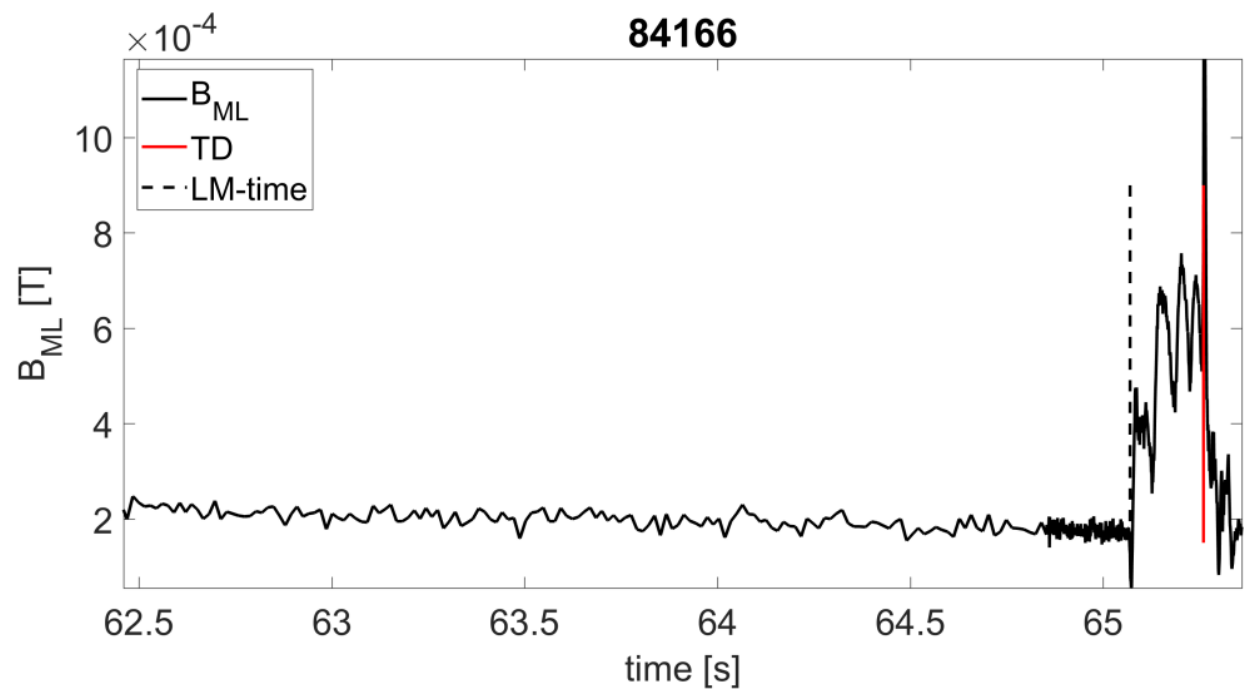

Figure 3. Example of locked mode raw signal of a JET disrupted pulse. The vertical dashed line indicates the LM time manually selected. The red vertical line refers the disruption time automatically calculated by DIS_tool. 


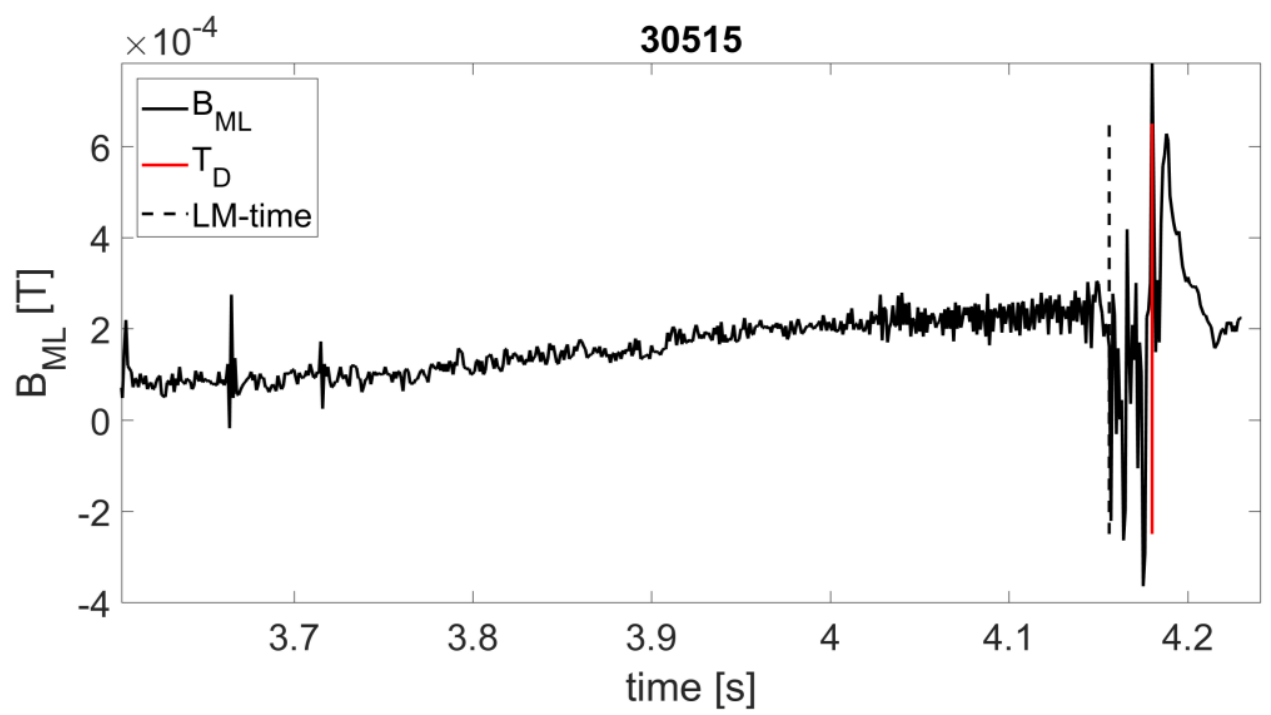

Figure 4. Example of locked mode raw signal calibrated in Tesla of an AUG disrupted pulse. The vertical dashed line indicates the LM time manually selected. The red vertical line refers the disruption time automatically calculated by DIS_tool.

Also for the non-disrupted pulses, the possible presence of locked mode corresponding to, e.g., minor disruptions, was manually verified and the locked mode time was recorded as well. Figure 5 shows a JET non-disrupted pulse where the locked mode signal suddenly increases in correspondence with a minor disruption (MD).

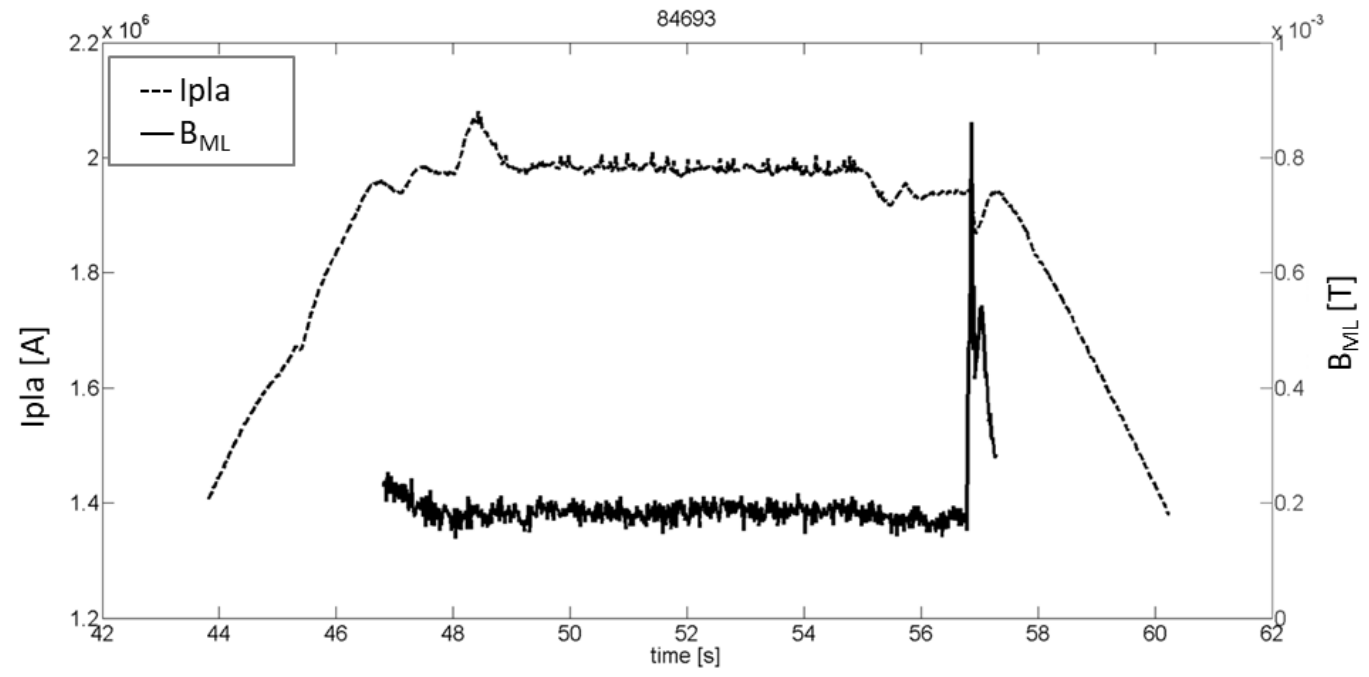

Figure 5. - Example of a JET non-disrupted pulse. The sudden increase of the locked mode raw signal corresponds to a minor disruption.

The results of the manual analysis are reported in Table 3. The data in the table helps to define the limits of a disruption predictor based only on the locked mode signal. Furthermore, a possible 
deterioration of the prediction performance passing from training to test sets and from JET to AUG can be quantified.

Table 3 -Results of the analyses of the locking of the mode in the databases of JET and AUG

\begin{tabular}{|c|c|c|c|c|c|c|c|c|}
\hline \multirow[b]{3}{*}{ Data set } & \multicolumn{4}{|c|}{ JET } & \multicolumn{4}{|c|}{ AUG } \\
\hline & & & & O-DISR & & & & O-DISR \\
\hline & No $L M$ & $\begin{array}{c}\text { LM } \\
\text { time } \\
\leq 10 \mathrm{~ms}\end{array}$ & LM Yes & $\begin{array}{c}\text { Corresponding } \\
\text { to MD }\end{array}$ & No LM & $\begin{array}{c}\text { LM } \\
\text { time } \\
\leq 2 m s\end{array}$ & LM Yes & $\begin{array}{c}\text { Corresponding } \\
\text { to } \mathrm{MD}\end{array}$ \\
\hline Training & $0 \%$ & $1.85 \%$ & $0 \%$ & $0 \%$ & $0 \%$ & $0 \%$ & $0 \%$ & $0 \%$ \\
\hline Test & $1.90 \%$ & $19.52 \%$ & $3.97 \%$ & $90 \%$ & $7.21 \%$ & $13.96 \%$ & $6.69 \%$ & $88.23 \%$ \\
\hline
\end{tabular}

By analysing the JET data, it follows that, in the disruptive training set, all the discharges have a locked mode, but, for $1.85 \%$ of them, the locked mode signal starts to rise less than 10ms before the disruption. According to [7], in JET a detection is considered tardy if the alarm is triggered less than $10 \mathrm{~ms}$ before the disruption time, where $10 \mathrm{~ms}$ is minimum time need by JET mitigation systems to intervene. The analysis of the disruption test set showed that for $1.90 \%$ of disruptions there is no locked mode, and for as much as $19.52 \%$ of them the locked mode signal starts to rise less than 10ms from disruption time, hence too late to allow even a mitigation action. This would result in a corresponding percentage of missed or tardy detections if a disruption predictor makes use of only the locked mode signal amplitude. Note that, if such a disruption predictor missed the alarm in these disrupted shots it does not make a mistake because it has to simply recognize the presence of the locked mode. In this paper, the shots where there is no rise in the locked mode signal will be not considered in the test set. It has to be noted that the non-disrupted pulses in the training set do not present locked modes, whereas in the $3.97 \%$ of non-disrupted pulses in the test set there is a locked mode and in $90 \%$ of them it corresponds to minor disruptions. This could produce false alarms in a locked mode based disruption predictor. These shots will not be considered in the evaluation of the predictor performance. In fact, in the framework of the analysis presented in this work, the prediction errors due to a not successful detection of a locked mode must not be confused with a not successful detection of a disruption with no locked mode, since the main objective of the proposed indicator is to maximize the detection of the locked mode. This shot selection does not affect the training set composition reported in Table 1; whereas, the test set, after removing 7 non-disruptive shots due to the lack of variables needed for the normalization, results in 206 disruptive shots and 234 non-disrupted shots in the test set. 
Referring to AUG, data in Table 3 shows that, in the disruption test set, $7.21 \%$ of pulses does not have locked mode whereas in $13.96 \%$ of them it locks too late, i.e., the LM-raw amplitude starts to rise less than $2 m s$ before $t_{D}$ (the minimum time needed by the AUG mitigation system to intervene is $2 m s$, in this case a disruption is tardy detected if the alarm is triggered later than $2 m s$ before the disruption time [11], [12]). Moreover, in the non-disrupted test set, $6.69 \%$ of pulses present a locked mode, $88.23 \%$ of them corresponding to minor disruptions.

The conclusions made for JET apply also for AUG: we may expect missed alarms, tardy detections and false alarms that cannot be avoided using only the amplitude of the locked mode raw signal. Also in the AUG case, disrupted discharges without locked mode and non-disrupted discharges where the locked mode is present have been excluded from the evaluation of the prediction performance. This selection does not affect the composition of the AUG training set reported in Table 3. Whereas the test set, after removing 13 disruptive and 2 non-disruptive shots due to the lack of variables needed for the normalization, results in 193 disruptive and 235 nondisrupted shots. Table 4 summarizes the composition of the database used to evaluate the prediction performances for the two machines.

Moreover, comparing the statistics resulting from the manual locked mode analysis made for the two devices, it could be expected that a predictor based on the LM raw signal has worst performance on AUG than on JET. The dissimilarities between the two devices have been discussed in [3].

Table 4 -resulting number of shots for JET and AUG

\begin{tabular}{|c|c|c|c|c|}
\hline \multirow{2}{*}{ Data set } & \multicolumn{2}{|c|}{ JET } & \multicolumn{2}{c|}{ AUG } \\
\cline { 2 - 5 } & DISR & NO-DISR & DISR & NO-DISR \\
\hline Training & 54 & 84 & 60 & 72 \\
\hline Test & 206 & 234 & 193 & 235 \\
\hline
\end{tabular}

\section{Locked Mode signal for disruption prediction}

Presently, on JET and AUG, the protection against disruptions uses the amplitude of the locked mode signal as a trigger. In particular, the alarm threshold is set on the $B_{M L} / I$ pla signal in [mT/MA] at JET and on the LM-raw signal in [V] at AUG. Hence, there is no correlation between the two trigger values. In a view of a machine independent disruption prediction 
approach, dissimilarities between the two locked mode triggers can be overcome taking into account, for both machines, the raw locked mode signal calibrated in Tesla $\left(B_{M L}\right)$ and normalized with respect to $B_{\vartheta}(0, a)$. The suitability of the proposed normalized signal as machine independent predictor will be confirmed if a common alarm threshold can be set for the two machines. To this aim, the distribution of the $B_{M L} / B_{\vartheta}(0, a)$ values in the pre-locking phase for JET and AUG have been compared. Figure 6.a reports such distribution for AUG in red, and for JET in blue. Note that, even if the post-locking phase of the locked mode signal is characterized by a disruptive behaviour, it has not been taken into account in this study because, once the trigger is activated, the values assumed in this post-locking phase are no longer relevant. As it can be noted, the two distributions in Figure 6.a show a common value that identifies the end of the pre-locking phase. For the sake of comparison, Figure 6.b reports the same distributions of the raw $B_{M L}$ values (the absolute values have been considered because, on AUG, the locked mode signal can assume positive and negative values). As it can be seen, in this case, a common value that identifies the end of the pre-locking phase cannot be found.

In this paper, the value that identifies the end of the pre-locking phase has been defined as that corresponding to the 99.7 percentile. Table 5 reports the values of the two signals at 99.7 percentile for JET in the first column, and for AUG in the second column. The remaining $0.3 \%$ of values has been, indeed, considered as outliers due to spikes and other short transient phenomena. Moreover, in order to appraise the similarity between these "thresholds" on the two machines, the third column of Table 5 reports the percentage deviation of these two values with respect their mean value. As can be seen, for $B_{M L}$, the threshold values of $0.311 \mathrm{mT}$ for JET and of $0.239 \mathrm{mT}$ for AUG are found, with a deviation of $13.09 \%$, whereas, for $B_{M L} / B_{9}(0, a)$, the threshold values of $0.69910^{-3}$ for JET and of $0.70010^{-3}$ for AUG are found, with a negligible deviation of $0.07 \%$. 

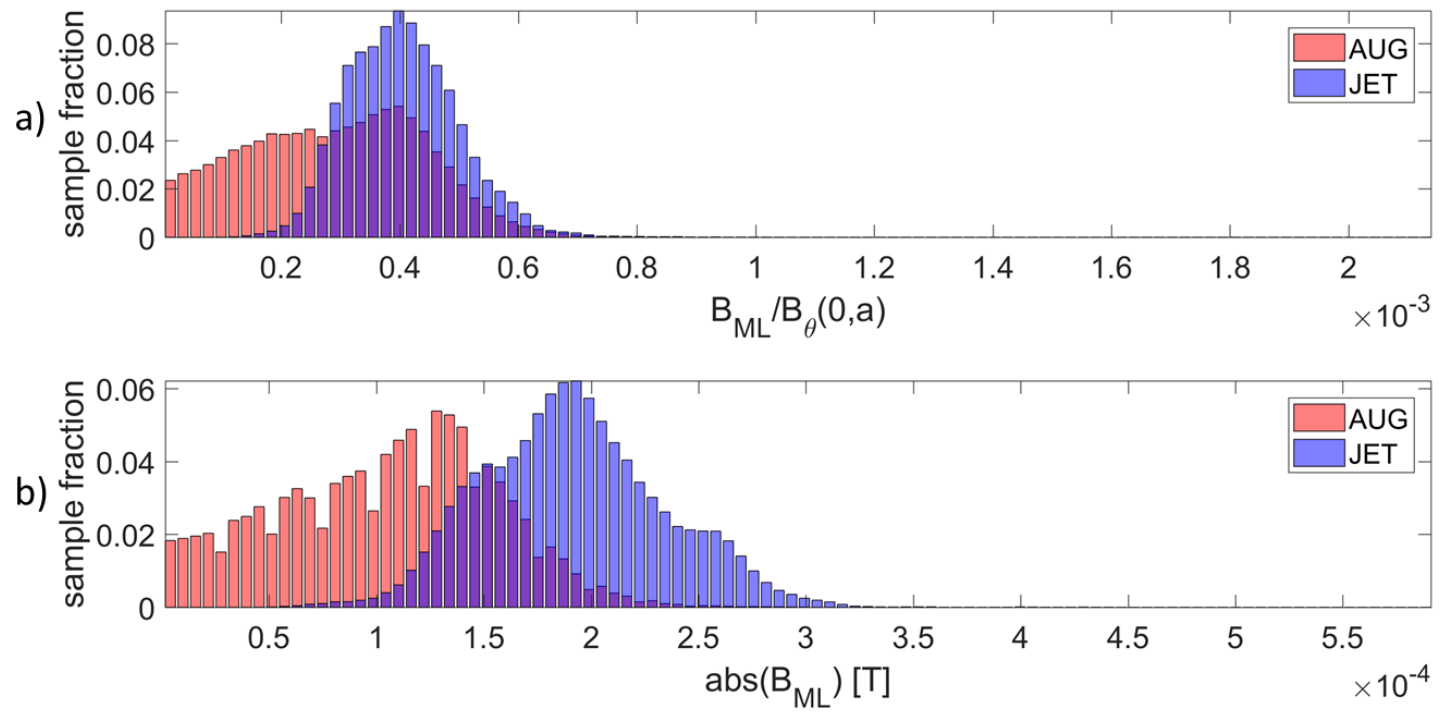

Figure 6. a) Distribution of the $B_{M L} / B_{\vartheta}(0, a)$ values in the pre-locking phase for JET (blue) and AUG (red) disruptive shots; b) Distribution of the absolute values of $B_{M L}[\mathrm{~T}]$ in the pre-locking phase for JET (blue) and AUG (red).

Table 5. Threshold values at 99.7 percentile

\begin{tabular}{lccc}
\hline Signal & JET & AUG & Deviation\% \\
\hline$B_{M L}$ & $3.1110^{-4}$ & $2.3910^{-4}$ & 13.09 \\
$B_{M L} / B_{\vartheta}(0, a)$ & $6.9910^{-4}$ & $7.0010^{-4}$ & 0.07 \\
\hline
\end{tabular}

Hence, the proposed normalized signal $B_{M L} / B_{\vartheta}(0, a)$ allows us to set a common value of about $0,7 \cdot 10^{-3}$ as upper bound of the pre-locking values for both the machines. Then, in order to set a common alarm threshold for $B_{M L} / B \vartheta(0, a)$ for disruption prediction purposes, an heuristic optimization procedure has been performed by minimizing the prediction error (Error) defined as the weighted sum of the false alarms, missed alarms and tardy predictions, as reported in (7) :

$$
\text { Error }=\frac{n_{D} \cdot(M A+T D)+n_{N D} \cdot F A}{n_{D}+n_{N D}}
$$

where $n_{D} \cdot$ and $n_{N D}$ are the number of disruptive and non-disruptive shots; $F A$ is the number of false alarms, $M A$ is the number of missed alarms, $T D$ is the number of tardy detections.

In this case, the training set is the combination of the training sets of the two machines. Defining the warning time as the difference between the disruption time $t_{D}$ and the alarm time given by the predictor, for a disruptive shot, the detection is considered successful (SP) if the warning time is longer than $10 m s$ for JET (it becomes $2 m s$ for AUG), otherwise it is tardy; whereas the alarm is 
missed if the disruption is not detected at all. For a non-disruptive pulse the false alarm occurs when it is detected as disruptive. Figure 7 reports, in green, the prediction error as a function of the threshold values on $B_{M L} / B_{\vartheta}(0, a)$ for the combined training set. An optimal threshold value of $0.73710^{-3}$, corresponding to the minimum of this error function, is obtained resulting in an overall Error of $9.63 \%$ for the combined training set. The same Figure 7 reports, in blue, the prediction error for the training sets of JET and, in red, for that of AUG. Using the previously optimized threshold, an Error of $5.07 \%$ for the JET training set and of $14.39 \%$ for the AUG training set is obtained.

Figure 8 reports the prediction errors as a function of the threshold values on $B_{M L}$ for the combined training set (green), for the JET training set (blue), and for the AUG training set (red). A threshold value of $0.326 \mathrm{mT}$, which minimizes the prediction error on the combined training set, results in an overall Error of $10.37 \%$, producing an Error of 3.62\% for JET, and bringing up to $17.42 \%$ on the AUG training set. Moreover, Figure 8 clearly shows that, to improve the performance of AUG, a lower threshold value has to be chosen to the detriment of the JET performance: the prediction error on AUG can be reduced to $12.88 \%$ with a threshold of 0.272 $\mathrm{mT}$, corresponding to an error of $21.01 \%$ for JET (the prediction error on the combined training set will be equal to $17.04 \%$ ).

This analysis shows, first of all, that both $B_{M L}$ and $B_{M L} / B_{\vartheta}(0, a)$ used as disruption predictors perform better for JET than for AUG. Moreover, it demonstrates that, for $B_{M L} / B_{\vartheta}(0, a)$, a shared alarm threshold can be set getting a good compromise between the performance on the two machines. Therefore, $B_{M L} / B_{\vartheta}(0, a)$ is suitable to be used as a machine independent predictor or as input to build an indicator that aims to be a machine independent parameter.

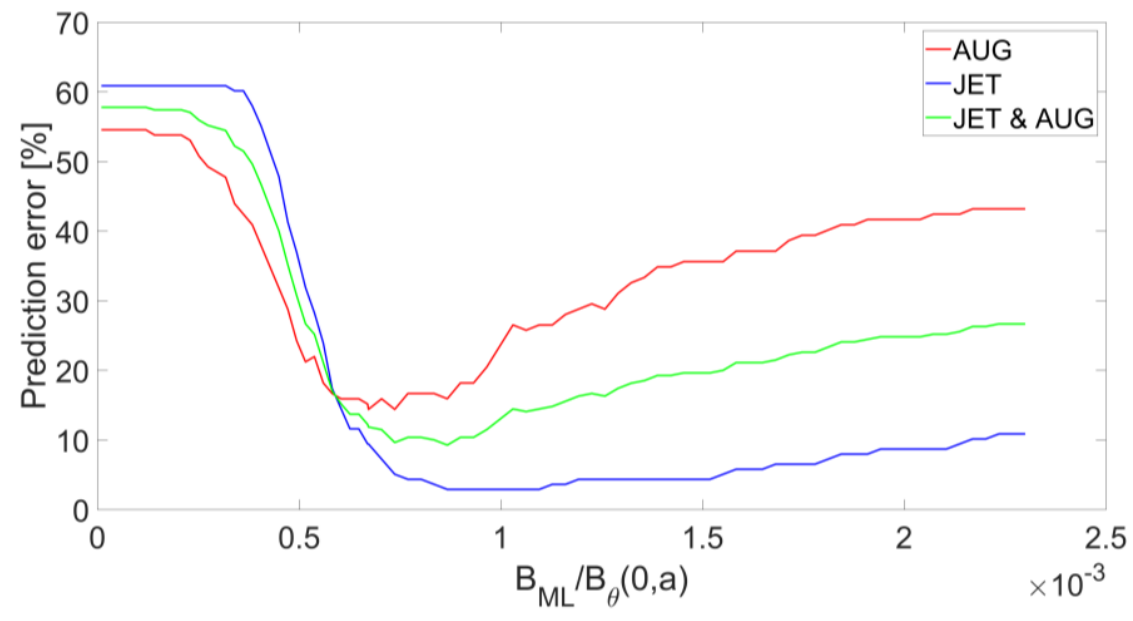


Figure 7. Prediction error as a function of the threshold values on $B_{M L} / B_{\vartheta}(0, a)$ for the training set of: AUG (red); JET (blue); JET and AUG (green).

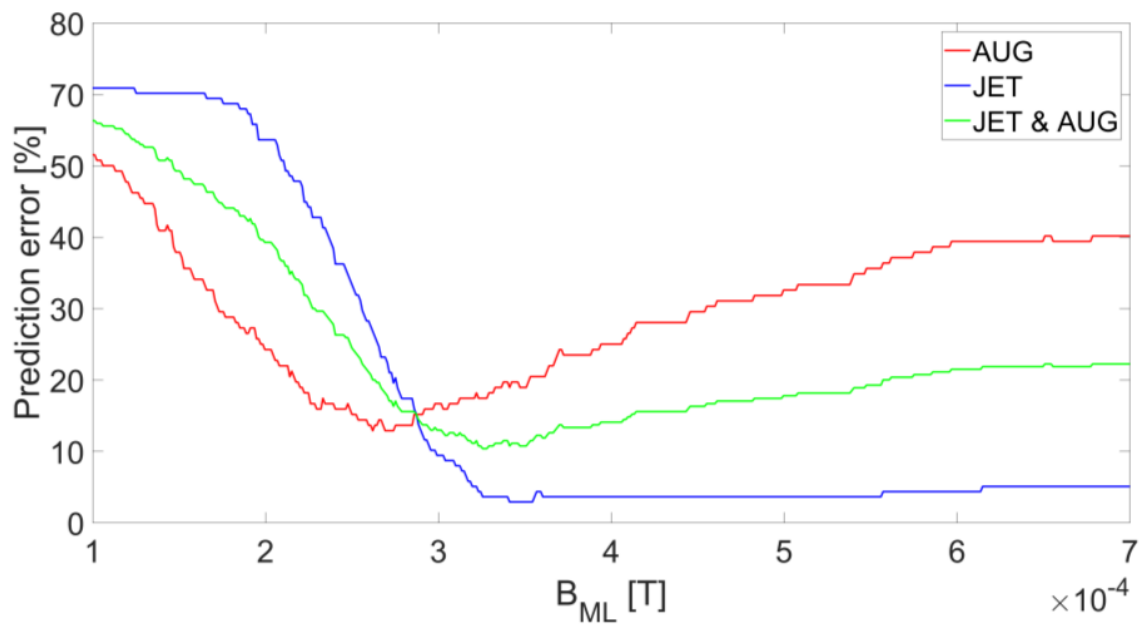

Figure 8. Prediction error as a function of the threshold values on $B_{M L}$ for the training set of: AUG (red); JET (blue); JET and AUG (green).

\section{Locked Mode Indicator as cross-machine disruption predictor}

The aim of the present study is to test the suitability of the proposed pre-processing algorithm to obtain a robust parameter to be used as input in a machine independent disruption predictor, possibly including other signals. To this end, the prediction capability of the proposed LM indicator has been evaluated and compared with respect to that of the normalized raw signal $B_{M L} / B_{\vartheta}(0, a)$.

In particular, an optimal threshold alarm value has been obtained also for $L M$-ind by minimizing, as in the previous section, the overall prediction error as a function of the threshold on the $L M$-ind. The first row in Table 6 reports the overall disruption prediction errors, i.e., the weighted sum of the false alarms, missed alarms and tardy predictions, obtained using a unique threshold on the $B_{M L} / B_{\vartheta}(0, a)$ signal for the training and the test set of JET and AUG, whereas the second row refers to the performance of $L M$-ind. 
Table 6. Comparison of the overall prediction error (Error) between $B_{M L} / B_{\vartheta}(0, a)$ signal and $L M$-ind for JET and AUG using a common threshold.

\begin{tabular}{lccccc}
\hline \multirow{2}{*}{ Signal } & Threshold & \multicolumn{2}{c}{ TRAINING } & \multicolumn{2}{c}{ TEST } \\
\cline { 3 - 6 } & & JET & AUG & JET & AUG \\
\hline$B_{M L} / B_{\vartheta}(0, a)$ & $7.37 \cdot 10^{-4}$ & $5.07 \%$ & $14.39 \%$ & $13.68 \%$ & $19.08 \%$ \\
LM-ind & $5.96 \cdot 10^{-16}$ & $2.90 \%$ & $5.30 \%$ & $10.60 \%$ & $14.92 \%$ \\
\hline
\end{tabular}

Table 6 reports also the selected optimal thresholds, common for the two machines. As can be noted, the overall error of $L M$-ind is smaller for both the machines even if the relative increase of the error between the training and the test at $A U G$ is more favourable for $B_{M L} / B_{9}(0, a)$.

\section{Machine-dependent Locked Mode disruption predictor}

The prediction performance of $L M$-ind can be further improved if different thresholds are optimized for each device. Regarding JET, Table 7 reports the prediction performance of both $B_{M L} / B_{\vartheta}(0, a)$ and $L M$-ind for the training and test sets in terms of successful predictions, missed alarms, and tardy detections on disruptive shots, and false alarms on non-disrupted shots. The last column reports the overall error (Error) on the training or test sets.

Table 7. Comparison of disruption prediction performance between $B_{M L} / B_{\vartheta}(0, a)$ signal and $L M$-ind for JET using a machine-dependent optimized threshold.

\begin{tabular}{|c|c|c|c|c|c|c|}
\hline \multirow{3}{*}{ Signal } & \multirow{3}{*}{ Threshold } & \multicolumn{5}{|c|}{ TRAINING SET } \\
\hline & & \multicolumn{3}{|c|}{ DISR } & \multirow{2}{*}{$\begin{array}{c}\text { NO-DISR } \\
\text { FA } \%\end{array}$} & \multirow{2}{*}{$\begin{array}{c}\text { TOT } \\
\text { Error \% }\end{array}$} \\
\hline & & SP \% & TD \% & MA \% & & \\
\hline$B_{M L} / B_{\vartheta}(0, a)$ & $8.80810^{-4}$ & 92.54 & 1.85 & 5.56 & 0 & 2.90 \\
\hline \multirow[t]{2}{*}{$L M$-ind } & $3.91110^{-17}$ & 96.30 & 1.85 & 1.85 & 1.19 & 2.17 \\
\hline & & \multicolumn{5}{|c|}{ TEST SET } \\
\hline \multirow[t]{2}{*}{ Signal } & \multirow[t]{2}{*}{ Threshold } & \multicolumn{3}{|c|}{ DISR } & NO-DISR & TOT \\
\hline & & SP \% & TD\% & MA \% & FA \% & Error \% \\
\hline$B_{M L} / B_{\vartheta}(0, a)$ & $8.80810^{-4}$ & 80.10 & 5.83 & 14.08 & 3.42 & 11.14 \\
\hline$L M$-ind & $3.91110^{-17}$ & 84.95 & 4.85 & 10.19 & 2.56 & 8.41 \\
\hline
\end{tabular}

As expected, the performance of both the normalized raw signal and the LM indicator deteriorates moving from training to test sets $\left(L M\right.$-ind and $B_{M L} / B_{\vartheta}(0, a)$ performances deteriorate of about 6 and 8 percentage points respectively). Nevertheless, LM-ind presents better 
performance with respect to $B_{M L} / B_{\vartheta}(0, a)$ in terms of successful prediction both in the training and in the test set, even if of few percent.

For the sake of comparison, an alarm threshold optimization procedure of the signal actually used as disruption prediction at JET $\left(B_{M L} / I_{p l a}\right)$ has been performed on the same database; on the test set it achieves a total error of $11,6 \%$, resulting in $78,6 \%$ of correct disruption predictions and $3 \%$ of false alarms.

In the following section, a deep analysis of the possible causes of the prediction errors has been done in order to separate the intrinsic limits of the raw signal from that of the considered predictors.

Figure 9 reports the cumulative warning time distribution for JET test set for $B_{M L} / B_{\vartheta}(0, a)$ and $L M$-ind (in blue and green respectively). The cumulative warning time distribution is defined as the fraction of correctly detected disruptions for which the warning time takes values larger than or equal to the considered one.

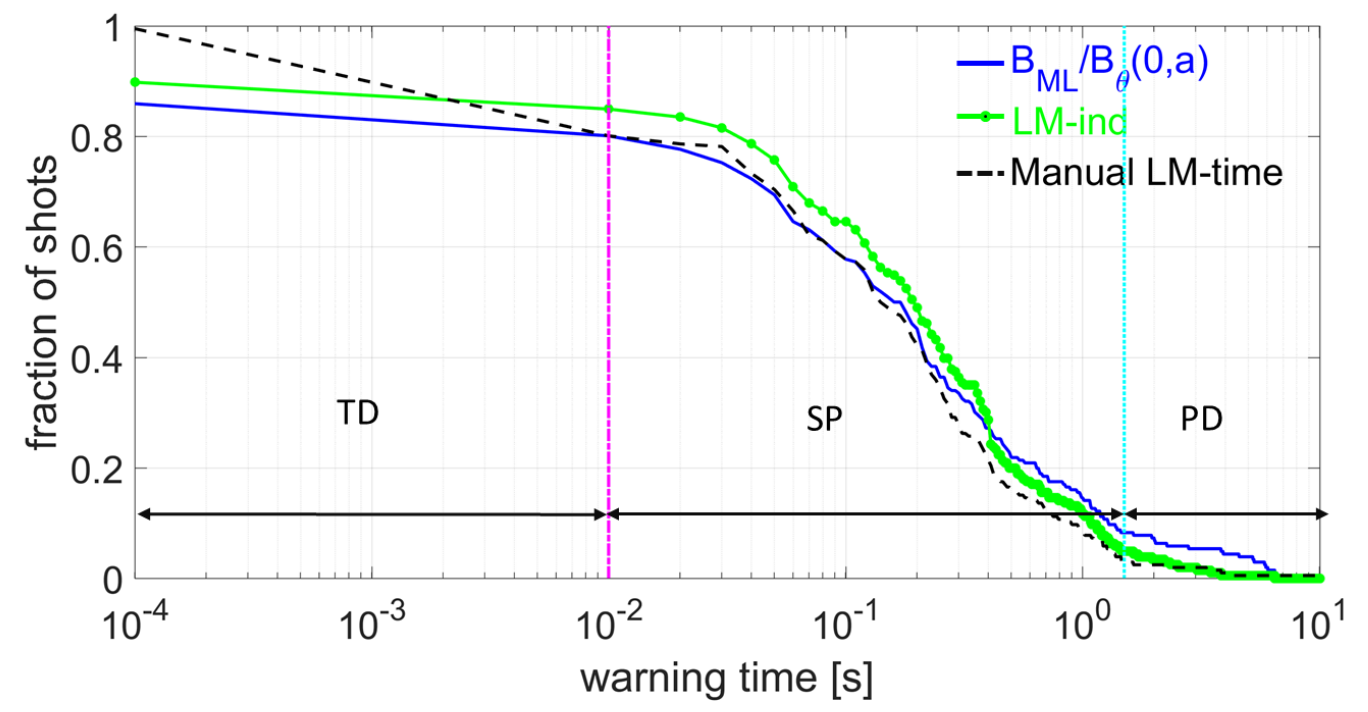

Figure 9. Cumulative warning time distribution for the test set of JET (TDs: tardy detections; SPs: successful predictions; PDs: premature detections).

In order to assess the relative performance of the two signals, they are compared with respect to the reference cumulative warning time distribution defined as the difference between the disruption time and the manually selected locked mode time ( $\mathrm{t}_{\mathrm{D}}$-LM-time), (dashed black line). It can be noted that the $L M$-ind obtains better results than $B_{M L} / B_{\vartheta}(0, a)$, anticipating the correct predictions and, at the same time, limiting the premature detections. Indeed, according to [7], 
setting the limit between correct and premature alarms at $1.5 \mathrm{~s}$ before the disruption time (cyan vertical dashed line in Figure 9), the achieved percentage of premature detections (PDs) are equal to $4.85 \%$ for $L M$-ind and $8.25 \%$ for $B_{M L} / B_{\vartheta}(0, a)$ on the test set. Note that, from the plot of Figure 9, the percentage of the tardy detections can be also obtained depending on its definition (dashed magenta line).

Table 8 , similar to Table 7 , refers to the prediction performance obtained by the normalized raw signal and by the LM indicator on the AUG database. As for JET, also for AUG the performance of both $B_{M L} / B_{\vartheta}(0, a)$ and $L M$-ind deteriorates going from training to test set (in this case $L M$-ind performance deteriorates of about 10 percentage points whereas the normalized raw signal deteriorates of 6 percentage points). However, $L M$-ind still shows an improvement of the overall performance of about $5 \%$ with respect to $B_{M L} / B_{\vartheta}(0, a)$.

For the sake of comparison, for AUG too, the performance of the signal presently used on the machine as disruption prediction (LM-raw) has been evaluated on the same database. Thus, an alarm threshold optimization procedure has been carried out on LM-raw, obtaining an overall error on the test set of $16.44 \%$, corresponding to $83,07 \%$ of successful predictions and $16 \%$ of false alarms.

Table 8. Comparison of disruption prediction performance between $B_{M L} / B_{\vartheta}(0, a)$ and $L M$-ind for AUG using a machine-dependent optimized threshold.

\begin{tabular}{lcccccc}
\hline & & & \multicolumn{3}{c}{ TRAINING SET } \\
\cline { 3 - 7 } Signal & Threshold & & DISR & & NO-DISR & TOT \\
\cline { 3 - 7 } & & SP \% & TD \% & MA \% & FA \% & Error \% \\
\hline$B_{M L} / B_{\vartheta}(0, a)$ & $7.48710^{-4}$ & 85.00 & 5.00 & 10 & 12.50 & 13.64 \\
$L M$-ind & $4.75410^{-16}$ & 93.33 & 1.67 & 5.00 & 1.39 & 3.79 \\
\hline & & & & TEST SET & & TOT \\
\cline { 3 - 7 } Signal & & & DISR & & NO-DISR & Error \% \\
\cline { 3 - 7 } & $7.48710^{-4}$ & 70.98 & 11.40 & 17.62 & 11.91 & 19.63 \\
\hline$B_{M L} / B_{\vartheta}(0, a)$ & $4.75410^{-16}$ & 78.24 & 10.88 & 10.88 & 8.09 & 14.25
\end{tabular}

Figure 10 reports the cumulative warning time distribution of $B_{M L} / B_{\vartheta}(0, a), L M$-ind and the manually selected LM-time on the AUG test sets. It should be noted that LM-ind obtains better results than $B_{M L} / B_{\vartheta}(0, a)$, anticipating the correct predictions and, at the same time, limiting the premature detections. It can be noted that there is an inversion of such trend starting at $30 \mathrm{~ms}$ and 
back in time until the 500ms. According to [11] [12], 500ms before the disruption time has been set as the limit between correct and premature alarms (cyan vertical dashed line); accordingly to this definition the percentages of premature detections (PDs) are equal to $11.92 \%$ for $L M$-ind and $16.06 \%$ for $B_{M L} / B_{\vartheta}(0, a)$ on the test set.

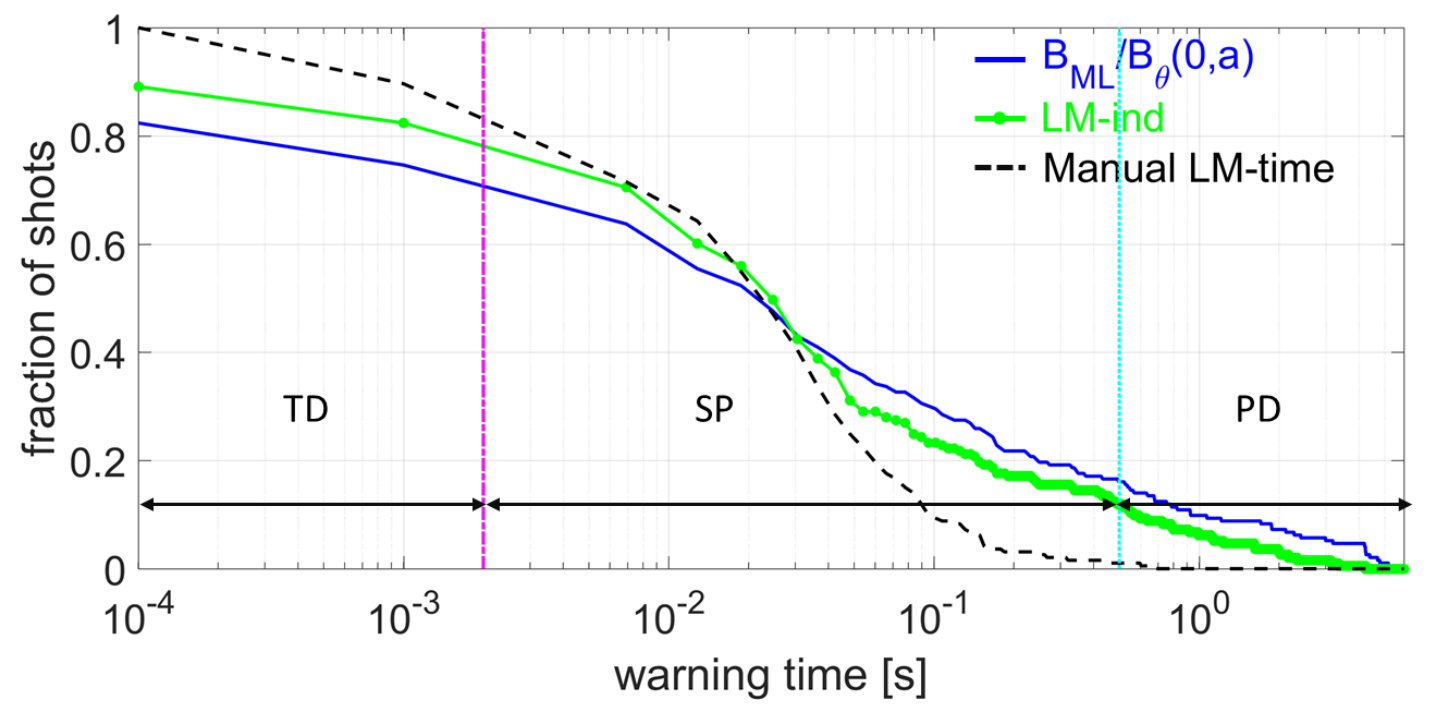

Figure 10. Cumulative warning time distribution for the test set of AUG (TDs: tardy detections; SPs: successful predictions; PDs: premature detections).

\section{Extrapolation to next-step fusion devices}

The predictions of plasma parameters to next-step fusion devices, such as ITER, can be obtained by extrapolation or interpolation from present day devices when larger databases are available. Since the alarm threshold values on LM-ind have been optimized on a large amount of data, coming from both JET and AUG, the extrapolation capability of the algorithm as predictor for a larger scale machine should be investigated. In this paper, this has been performed by using the $L M$-ind alarm threshold optimized for the smaller device (AUG) on the database of the larger one (JET). Table 9 reports the prediction performance obtained by $L M$-ind and $B_{M L} / B_{\vartheta}(0, a)$ on the JET database using the alarm threshold optimized on the AUG training set. As expected, the performances of both predictors are worse than those obtained with their own threshold optimized on the JET training set, but the results are anyway quite encouraging for both indicators. 
Table 9. Comparison of disruption prediction performance between $B_{M L} / B_{\vartheta}(0, a)$ signal and $L M$-ind for JET using the AUG optimized threshold.

\begin{tabular}{lcccccc}
\hline \multirow{2}{*}{ Signal } & \multirow{5}{*}{ Threshold } & \multicolumn{5}{c}{ TRAINING SET } \\
\cline { 3 - 7 } & & SP \% & TD \% & MA \% & FA \% & Error \% \\
\cline { 3 - 7 } & & 92.59 & 3.70 & 3.70 & 3.57 & 5.07 \\
\hline$B_{M L} / B_{\vartheta}(0, a)$ & $7.48710^{-4}$ & 3.70 & 3.70 & 0.00 & 2.90 \\
$L M$-ind & $4.75410^{-16}$ & 92.59 & & TEST SET & NO-DISR & TOT \\
\hline \multirow{2}{*}{ Signal } & \multirow{2}{*}{ Threshold } & & DISR & & NO-DISR & TOT \\
\cline { 3 - 7 } & & SP \% & TD\% & MA \% & FA \% & Error \% \\
\hline$B_{M L} / B_{\vartheta}(0, a)$ & $7.48710^{-4}$ & 82.52 & 3.88 & 13.59 & 10.26 & 13.64 \\
$L M$-ind & $4.75410^{-16}$ & 79.13 & 5.34 & 15.53 & 0.85 & 10.23 \\
\hline
\end{tabular}

\section{Analysis of prediction errors}

This section reports the results of the analysis performed to identify the causes of FAs, MAs, and TDs for both the normalized raw signal and the LM indicator. The following analysis shows that some intrinsic problems in the raw signal such as drifts, offsets, and error field pick up coils (EFCC), can cause false alarms and incongruous alarms, which are not related to physical reasons.

The analysis has been made making reference to the different predictions performed by the two considered predictors, $B_{M L} / B_{\vartheta}(0, a)$ and $L M$-ind. To this purpose, Table 10 and Table 11 report the comparison of the performance of $B_{M L} / B_{\vartheta}(0, a)$ and $L M$-ind on JET and AUG, respectively. In particular, each element in the tables refers to the performance (in terms of successful-disruptive prediction, tardy detections, missed alarms, successful predictions on regular terminations and false alarms) indicated at the corresponding row for $B_{M L} / B_{\vartheta}(0, a)$ and at the corresponding column for the $L M$-ind. The elements in the main diagonal are the number of pulses for which the two predictors provide the same prediction (successful or wrong), whereas the elements outside that diagonals contain the number of pulses for which the two predictors provide a different prediction. For example, the element in the $2^{\text {nd }}$ row and $1^{\text {st }}$ column of the tables represents the number of shots tardy detected by $B_{M L} / B \vartheta(0, a)$ that are successfully predicted by $L M$-ind, whereas the element in the $4^{\text {th }}$ row and $5^{\text {th }}$ column contains the number of false alarms of $L M$-ind that have been correctly recognized as non-disruptive shots by $B_{M L} / B_{\vartheta}(0, a)$. On the contrary the number in 
the $5^{\text {th }}$ row, $4^{\text {th }}$ column indicates how many non-disrupted pulses successfully predicted by $L M$-ind are detected as disruptive by $B_{M L} / B_{\vartheta}(0, a)$.

Hence, looking through the first row it is possible to read how many disruptions successfully predicted by $B_{M L} / B_{\vartheta}(0, a)$ are tardy detected $\left(2^{\text {nd }}\right.$ column) or missed ( $3^{\text {th }}$ column) by $L M$-ind. On the other hand, looking through the first column it is possible to read how many disruptions successful predicted by $L M$-ind are tardy detected ( $2^{\text {nd }}$ raw) or missed ( $3^{\text {th }}$ raw) by $B_{M L} / B_{\vartheta}(0, a)$.

\section{Analysis of prediction errors at JET}

Table 10 reports the results related to the entire JET database (training and test). As expected, $L M$-ind works better than $B_{M L} / B_{\vartheta}(0, a)$ both on false alarms and tardy detections. In particular, the LM indicator recovers 4 TDs and 8 MAs of the $B_{M L} / B_{\vartheta}(0, a)$, whereas the $B_{M L} / B_{\vartheta}(0, a)$ does not recover any MAs or TDs of the $L M$-ind.

Table 10. Comparison of the prediction performance of $B_{M L} / B_{\vartheta}(0, a)$ and $L M$-ind on JET. The main diagonal reports the number of pulses for which the two predictors provide the same prediction; the elements outside the diagonals report the number of pulses for which the two predictors provide a different prediction.

\begin{tabular}{|c|c|c|c|c|c|c|}
\hline & & \multicolumn{5}{|c|}{ LM-ind } \\
\hline & & SPs-Disr & TDs & MAs & SPs NO-DISR & FAs \\
\hline \multirow{5}{*}{$\begin{array}{l}\bar{b} \\
\tilde{o} \\
0 \\
0 \\
0 \\
0\end{array}$} & SPs-Disr & 215 & 0 & 0 & - & - \\
\hline & TDs & 4 & 10 & 0 & - & - \\
\hline & MAs & 8 & 1 & 22 & - & - \\
\hline & SPs NO-DISR & - & - & - & 305 & 5 \\
\hline & FAs & - & - & - & 6 & 2 \\
\hline
\end{tabular}

Figure 11 reports one of the MAs recovered by the LM indicator. For this pulse the LM-time is at 46.166s (1 $\mathrm{ms}$ before the disruption time), LM-ind triggers a premature alarm at 43.767s $(2.4 \mathrm{~s}$ before the disruption but, as can be noted, it is due to the information carried out by the frequency content of the signal, which is neglected by the predictor based on $B_{M L} / B_{\vartheta}(0, a)$. It is to be highlighted that, in $100 \%$ of the 8 recovered MAs, the sampling frequency increases some hundreds of $m s$ before the disruption; for 7 of them that is the cause of the LM-ind recovering. Hence, the algorithm, making use of the combined time-frequency information, captures a behavior that is not due to physical reasons, despite what happened for the remaining one, which is correctly recovered. 


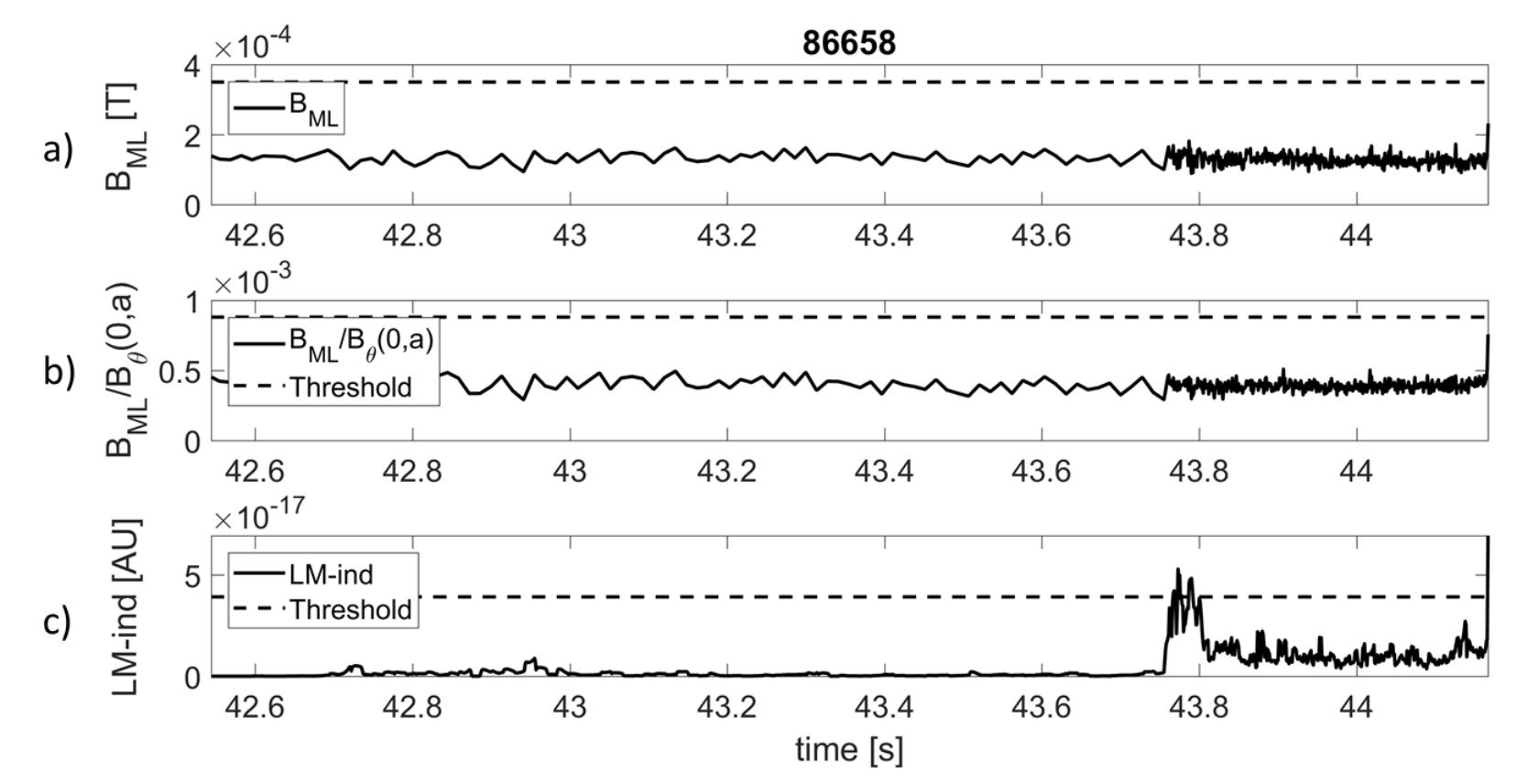

Figure 11. Example of a MA for $B_{M L} / B_{\theta}(0, a)$ due to late locking of the mode, recovered by the $L M$-ind in a JET pulse: (a) Locked Mode raw signal $B_{M L}$ in [T]; (b) Locked Mode raw signal normalized with respect to $B_{\theta}(0, a)$ in dimensionless units; (c) LM indicator in arbitrary units [AU]. The figure refers to the phase of the plasma current flat-top (from the X-point formation to $t_{D}$ ).

Note that, the sampling frequency can increase also in non-disrupted pulses, such as in the example reported in Figure 12. Because of the low value of the normalized raw signal amplitude, this effect does not trigger FAs. In particular, $14.96 \%$ of non-disrupted shots in the test set have at least one change in the sampling frequency, but $94.29 \%$ of them are correctly predicted by the $L M$-ind. Hence, weighting the amplitude of the frequency components with the inverse of their squared frequency has a beneficial effect of increasing the successful predictions and not affects the false alarm rate.

Figure 13 shows a zoom from a JET pulse, where the growth of the locked mode starts $20 \mathrm{~ms}$ before the disruption time and the amplitude of the normalized raw signal crosses the threshold $9 m s$ before the disruption time, causing a TD. On the contrary, the LM indicator algorithm, profiting of both amplitude and frequency content, is able to correctly trigger the alarm $17 \mathrm{~ms}$ before the disruption time. Only for one of the four recovered TDs, $L M$-ind improperly triggers the alarm due to the changing in the frequency content. 
a)
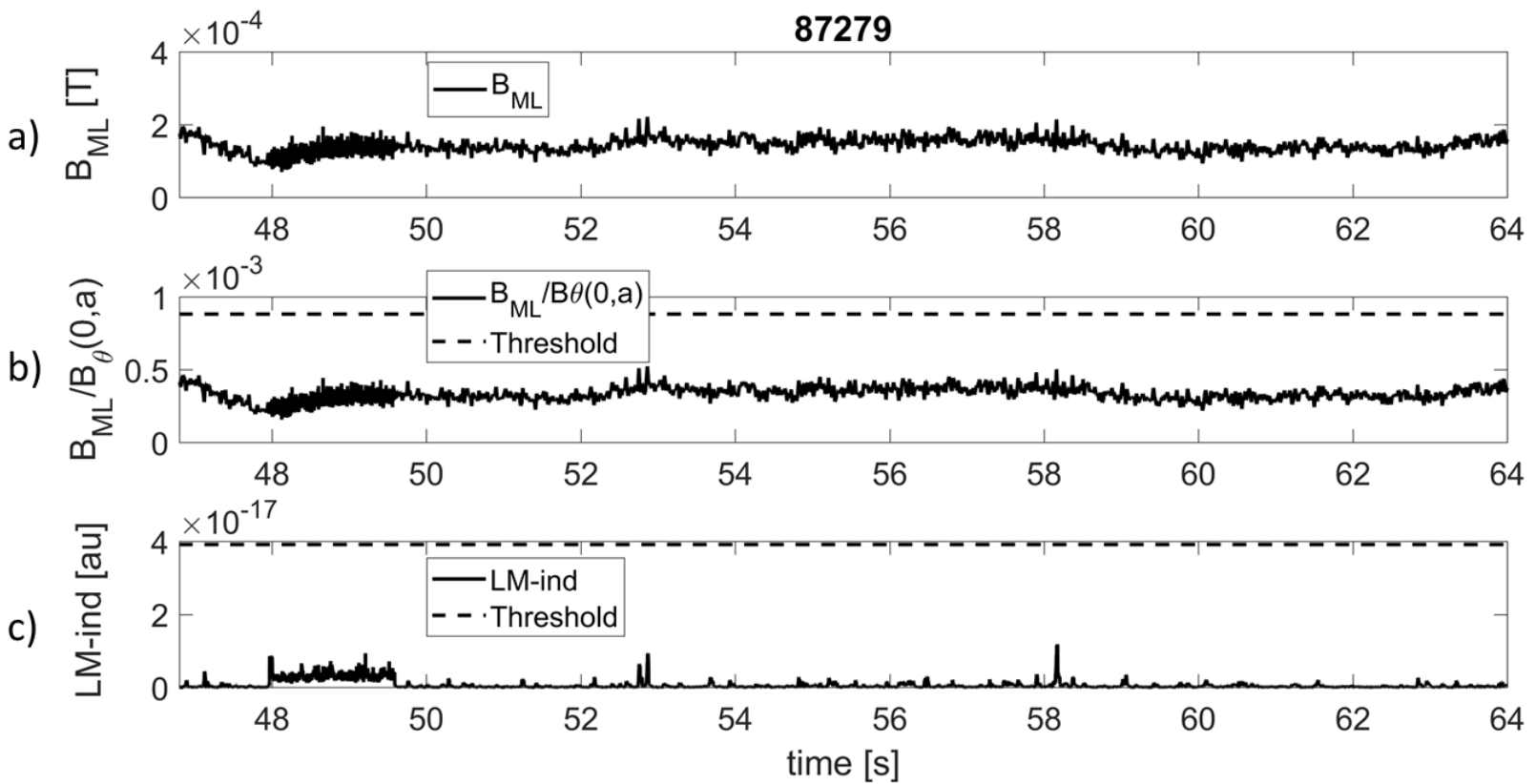

Figure 12. A JET non-disrupted pulse with a change in the sampling frequency: (a) Locked Mode raw signal $B_{M L}$ in [T]; (b) Locked Mode raw signal normalized with respect to $B_{\theta}(0, a)$ in dimensionless units; (c) LM indicator in arbitrary units [AU]. The figure refers to the phase of the plasma current flat-top.

a)

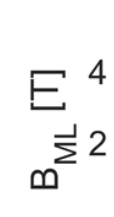

$$
\times 10^{-4}
$$
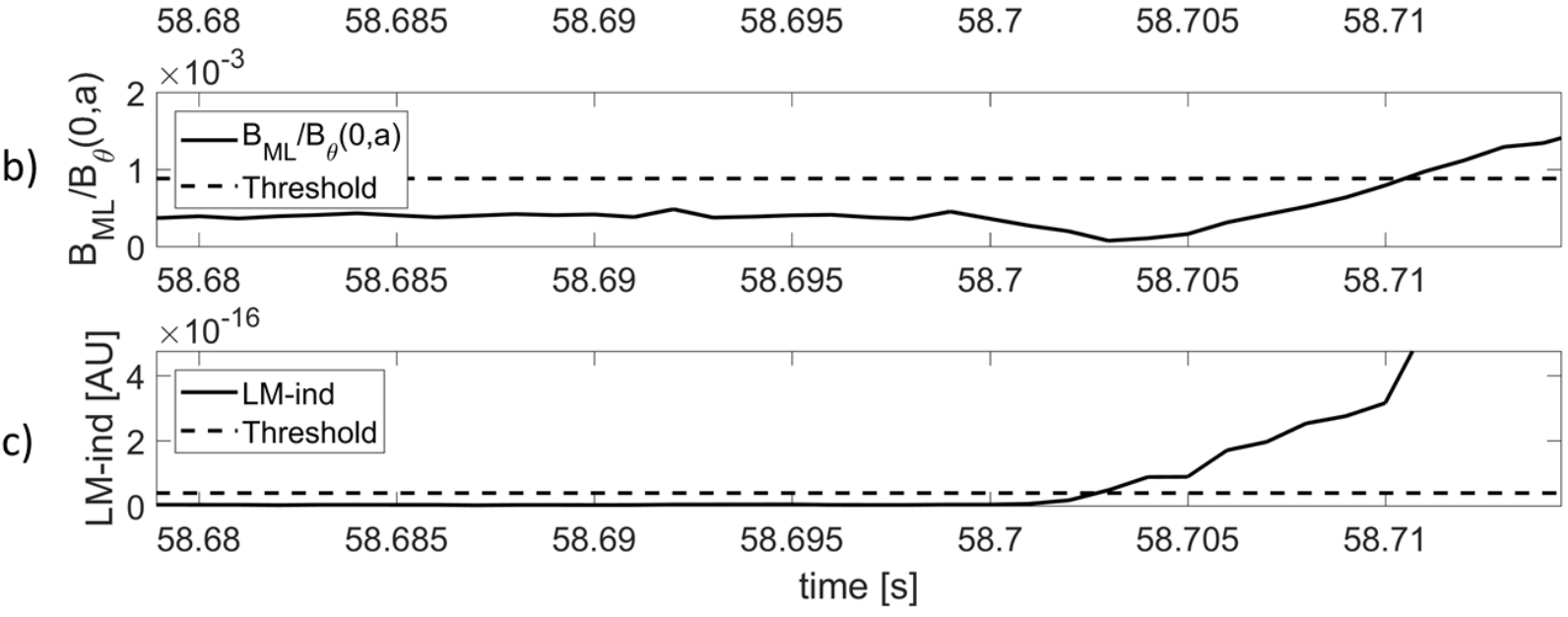

Figure 13. Example of a TD for $B_{M L} / B_{\theta}(0, a)$ recovered by the LM-ind in a JET disrupted pulse : (a) Locked Mode raw signal $B_{M L}$ in [T]; (b) Locked Mode raw signal normalized with respect to $B_{\theta}(0, a)$ in dimensionless units; (c) LM indicator in arbitrary units $[\mathrm{AU}]$. The figure refers to the last phase of the disruption up to $t_{\mathrm{D}}$. 
Concerning false alarms, $L M$-ind is able to recover 6 FAs of $B_{M L} / B_{\vartheta}(0, a)$, whereas $B_{M L} / B_{\vartheta}(0, a)$ recovers 5 FAs triggered by $L M$-ind. Figure 14 shows a JET pulse where no locked mode is present but, due to the offset of the raw signal (Figure 14.a), the normalized raw signal remains always above the optimized threshold causing a FA. On the contrary, the LM indicator algorithm removes this offset and no alarm is triggered.

a)

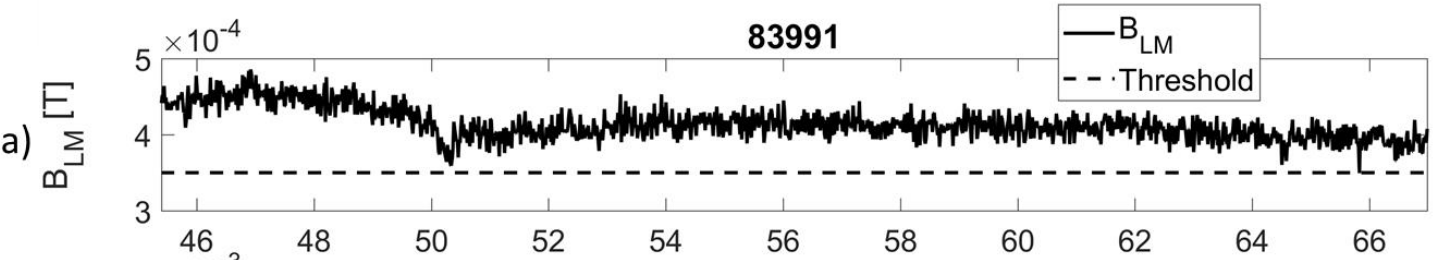

b)
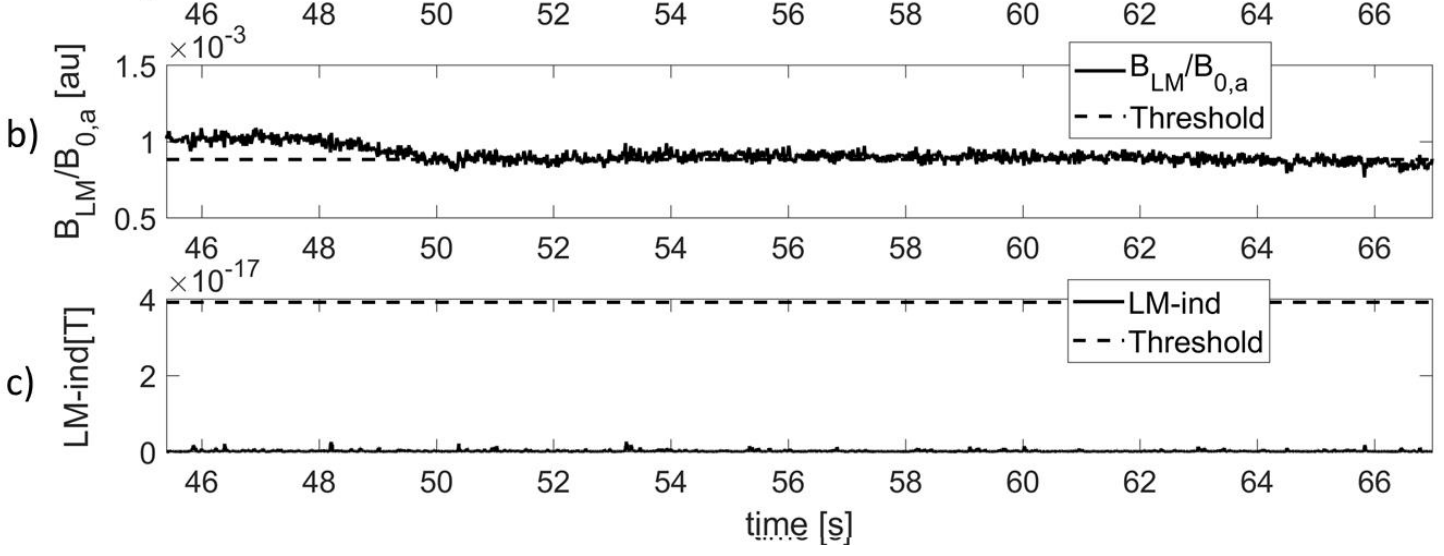

Figure 14. Example of a FA for $B_{M L} / B_{\theta}(0, a)$ recovered by the LM-ind in a JET non-disrupted pulse: (a) Locked Mode raw signal $B_{M L}$ in [T]; (b) Locked Mode raw signal normalized with respect to $B_{\theta}(0, a)$ in dimensionless units; (c) LM indicator in arbitrary units [AU]. The figure refers to the phase of the plasma current flat-top.

\section{Analysis of prediction errors at AUG}

The same analysis done for JET has been performed also for AUG, leading more or less to the same considerations. Table 11 shows the prediction performances made by $B_{M L} / B,(0, a)$ and $L M$-ind for the entire AUG database.

Table 11 shows that $L M$-ind works better than $B_{M L} / B_{\vartheta}(0, a)$ on missed alarms. In particular, the indicator recovers 19 MAs of $B_{M L} / B_{\vartheta}(0, a)$, whereas $B_{M L} / B_{\vartheta}(0, a)$ recovers 4 MAs of $L M$-ind. Actually, these 4 shots are erroneously successfully predicted by $B_{M L} / B_{\vartheta}(0, a)$ due to a drift and/or off-set on the raw signal. Figure 15 reports one of the four recovered MAs; in this pulse the locking of the mode occurs in the last two milliseconds before the disruption, but $B_{M L} / B_{\vartheta}(0, a)$ triggers the alarm well in advance due to an offset on the raw signal (see Figure 16.a). 
Table 11. Comparison of the prediction performance of $B_{M L} / B_{\vartheta}(0, a)$ and $L M$-ind on AUG. The main diagonal reports the number of pulses for which the two predictors provide the same prediction; the elements outside the diagonals report the number of pulses for which the two predictors provide a different prediction.

\begin{tabular}{|c|c|c|c|c|c|c|}
\hline & & \multicolumn{5}{|c|}{ LM-ind } \\
\hline & & SPs-Disr & TDs & MAs & SPs NO-DISR & FAs \\
\hline \multirow{5}{*}{$\begin{array}{l}\delta \\
\hat{e} \\
0 \\
0 \\
0 \\
0 \\
0\end{array}$} & SPs-Disr & 177 & 7 & 4 & - & - \\
\hline & TDs & 11 & 11 & 3 & - & - \\
\hline & MAs & 19 & 4 & 17 & - & - \\
\hline & SPs NO-DISR & - & - & - & 259 & 11 \\
\hline & FAs & - & - & - & 28 & 9 \\
\hline
\end{tabular}

a)

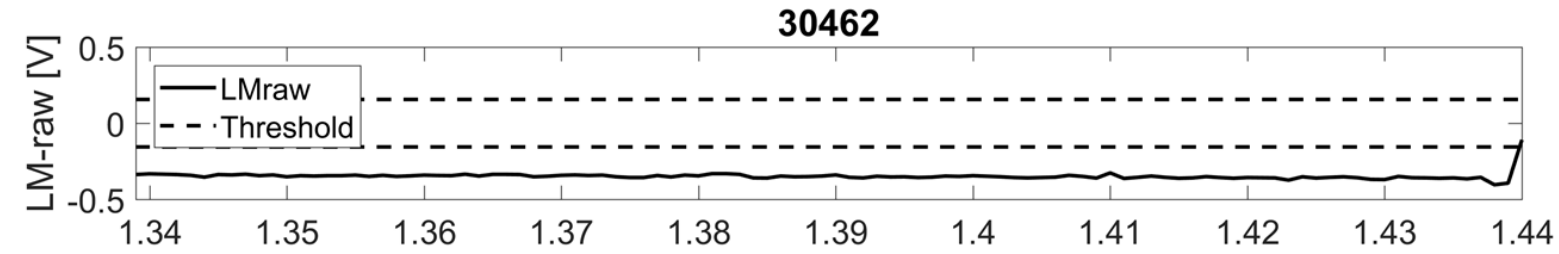

b)
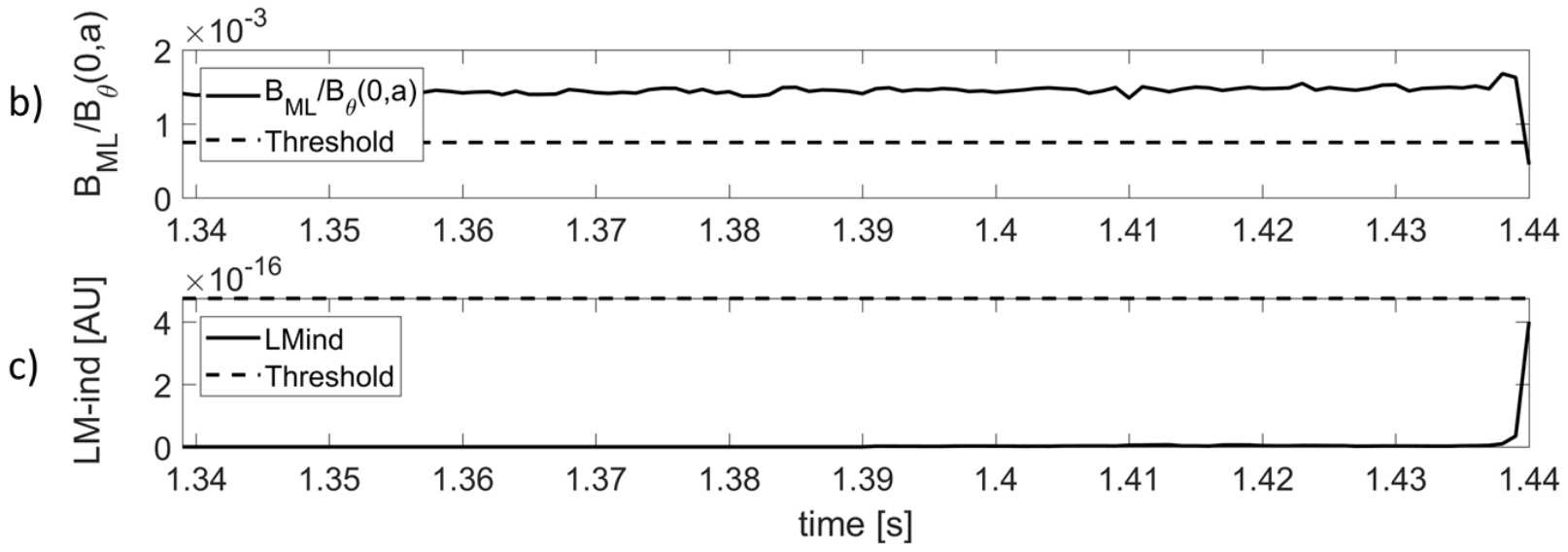

Figure 15. Example of an AUG missed alarm of the $L M$-ind, which is triggered by the $B_{M L} / B_{\vartheta}(0, a)$ signal due to the offset on the raw signal: (a) Locked Mode raw signal in [V]; (b) Locked Mode raw signal calibrated in [T] and normalized with respect to $B_{\theta}(0, a)$ in dimensionless units; (c) LM indicator in arbitrary units [AU]. The figure refers to the last phase of the disruption up to $t_{\mathrm{D}}$.

The LM indicator performs better than the normalized raw signal also regarding tardy detections: the indicator recovers 11 TDs of the $B_{M L} / B_{\vartheta}(0, a)$, whereas the $B_{M L} / B_{\vartheta}(0, a)$ recovers 7 TDs of the LM-ind. However, these last are incorrect alarms due to spikes, off-set and/or drift, such as for the disruptive pulse shown in Figure 16. For this shot the locking of the mode takes place $1 \mathrm{~ms}$ before the disruption time, and the alarm triggered by $B_{M L} / B_{\vartheta}(0, a)$ is due to a drift on the raw signal (see Figure 16.a). 
31292

a)
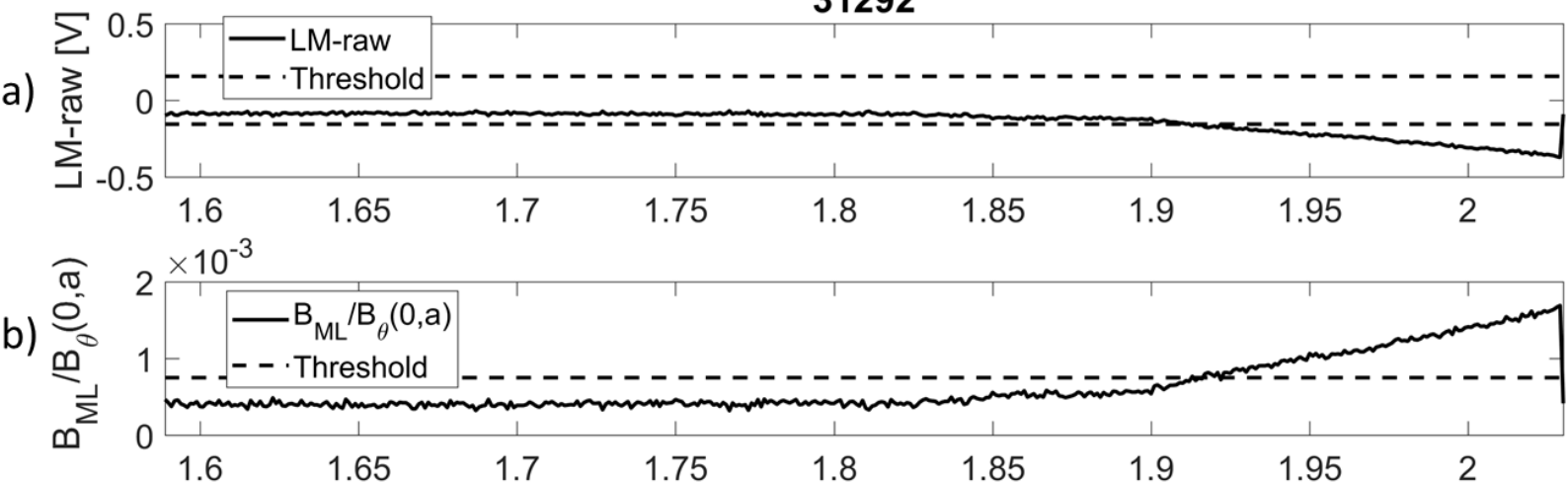

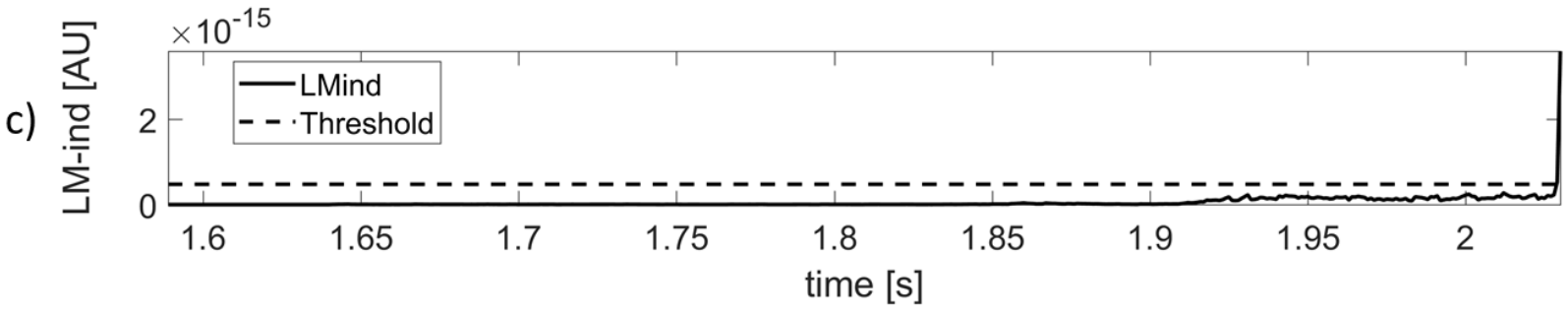

Figure 16. Example of AUG disruption predicted by the $B_{M L} / B_{\vartheta}(0, a)$ that is tardy detected by the $L M$-ind: (a) Locked Mode raw signal in [V]; (b) Locked Mode raw signal calibrated in [T] and normalized with respect to $B_{\theta}(0, a)$ in dimensionless units; (c) LM indicator in arbitrary units [AU]. The figure refers to the last phase of the disruption up to $t_{D}$.

Table 11 highlights that $L M$-ind outperforms $B_{M L} / B_{\vartheta}(0, a)$ also on false alarms recovering 28 false alarms of $B_{M L} / B_{\vartheta}(0, a)$, whereas $B_{M L} / B_{\vartheta}(0, a)$ recovers only 11 FAs of $L M$-ind. For 5 of these 11 FAs, LM-ind triggers an alarm in correspondence of a minor disruption as in the case shown in Figure 17. For the remaining 6 shots, the trigger of LM-ind is due to outliers or short transient phenomena as in the example reported in Figure 18. 
a)

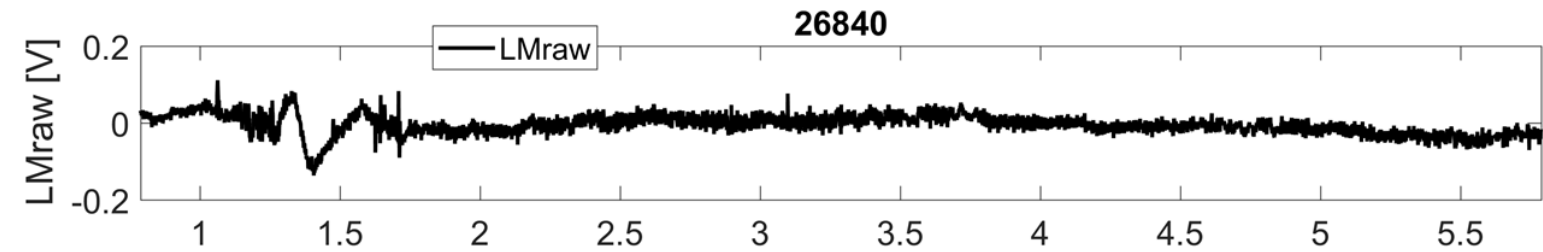

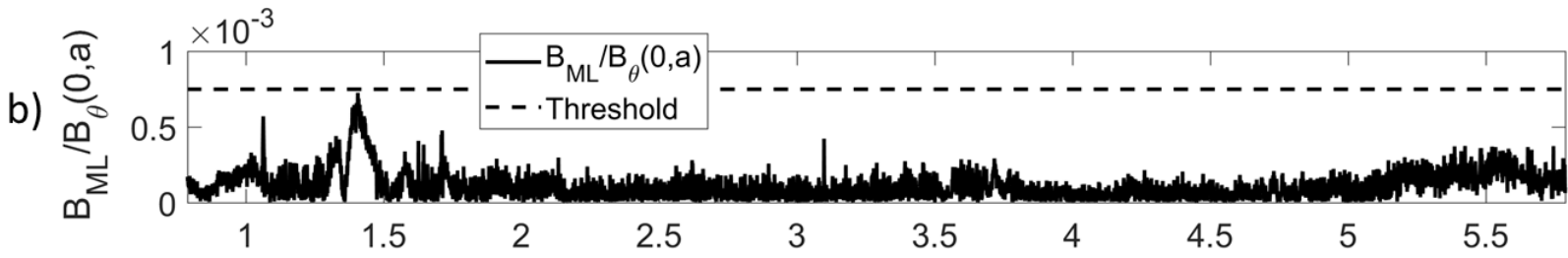

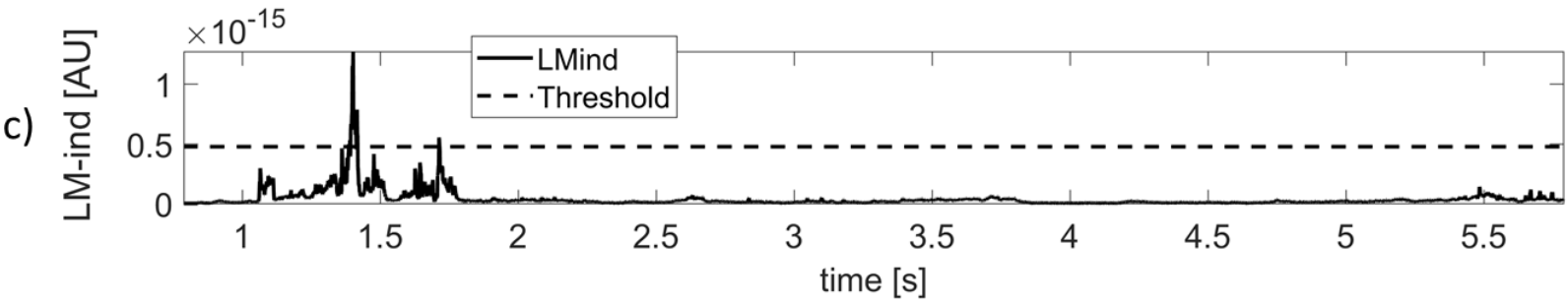

Figure 17. Example of a false alarm triggered by the $L M$-ind due to a minor disruption: (a) Locked Mode raw signal in [V]; (b) Locked Mode raw signal calibrated in [T] and normalized with respect to $B_{\theta}(0, a)$ in dimensionless units; (c) LM indicator in arbitrary units [AU]. The figure refers to the phase of the plasma current flat-top.

a)

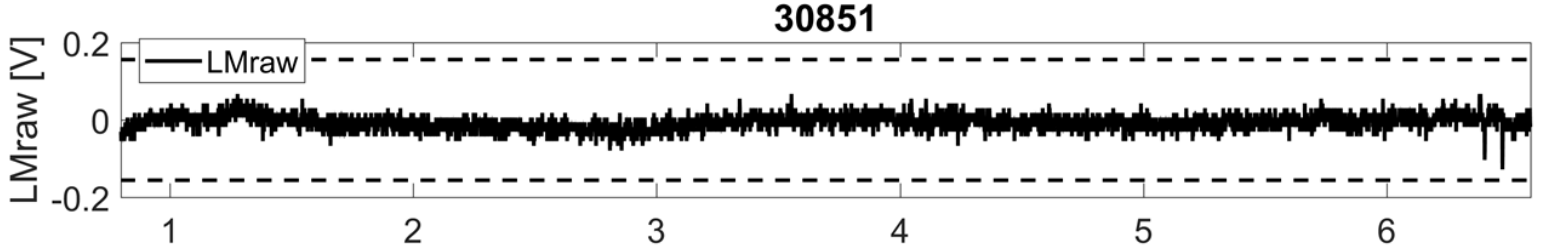

b)

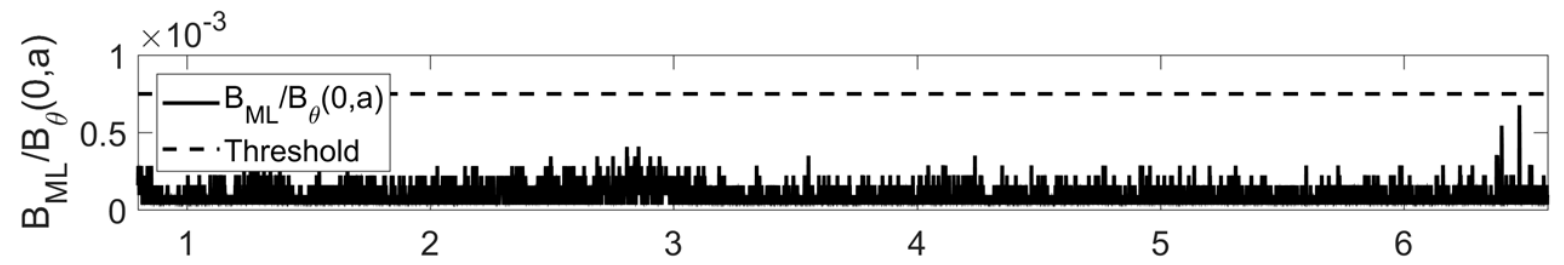

c)

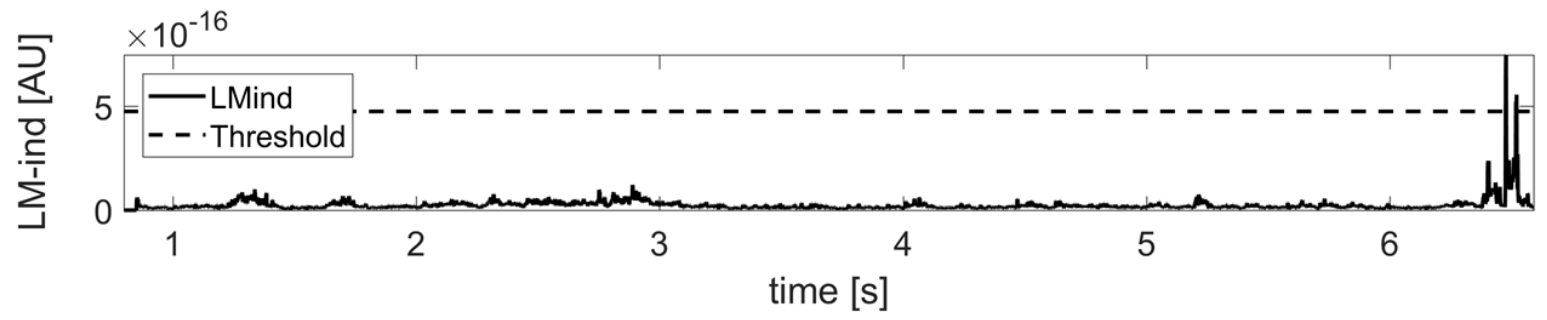

Figure 18. Example of a false alarm triggered by the LM-ind due to a spike: (a) Locked Mode raw signal in [V]; (b) Locked Mode raw signal calibrated in [T] and normalized with respect to $B_{\theta}(0, a)$ in dimensionless units; (c) LM indicator in arbitrary units $[\mathrm{AU}]$. The figure refers to the phase of the plasma current flat-top. 


\section{Conclusions}

The main aim of the paper was to evaluate the advantages and the limits of the proposed locked mode indicator if used in a disruption predictor instead of the raw signal, highlighting the possibility of improving the mode locking detection through a suitable processing algorithm exploiting both information in time and frequency domain. The paper, not only presents the performance of a locked mode based disruption predictor but also analyses the wrong prediction causes and investigates the suitability of the proposed algorithm for a multi-machine approach. This investigation could help to extend the proposed disruption prediction technique to the next generations of tokamaks, such as ITER.

Moreover, the present analysis wants to be a contribution toward the definition of robust indicators characterizing disruptive behaviours, and that could be scaled to different tokamaks. In particular, it has been shown that a unique alarm threshold can be found on different machines by using suitable scaled signals, when the considered devices are jointly involved in the training, a limited performance deterioration is observed with respect to machine customized thresholds. Indeed, a more consistent performance deterioration is achieved when the extrapolation capability of the algorithm, toward a larger scale machine, is investigated. From the analysis of the prediction errors it results that, both for JET and AUG disruptive shots, LM-ind recovers both MAs and TDs thanks to the frequency domain features. Moreover, it is able to correct inappropriate raw signal alarms triggered by intrinsic drift and offset problems thanks to detrending and off-set removal of the normalized LM amplitude. For the non-disrupted shots, $L M$-ind recovers FAs due to drift and/or offset.

Although prediction performances of the proposed indicator are quite good, and also better than those reported in the recent literature [7], the analyses show the intrinsic limits of a possible disruption predictor based on the unique locked mode signal. Future works will be focused on using the proposed LM indicator together with other plasma features as inputs of a multi-signal disruption prediction system, optimised in terms of success rate, warning times and that be differentiated depending on different disruptions classes.

\section{Acknowledgement}

This work has been carried out within the framework of the EUROfusion Consortium and received funding from the EURATOM research and training programme 2014-2018 under grant 
agreement No 633053. The views and opinions expressed herein do not necessarily reflect those of the European Commission.

\section{References}

[1] de Vries P.C., Johnson M.F., Alper B., Buratti P., Hender T.C., Koslowski H.R., Riccardo V. and JET-EFDA Contributors 2011, Survey of disruption causes at JET, Nucl. Fusion 51 53018.

[2] P.C. de Vries, et al., The influence of an ITER-like wall on disruptions at JET, Phys. Plasmas 21 (2014) 056101.

[3] De Vries P., et al 2016, Scaling of the MHD Perturbation Amplitude Required to Trigger a Disruption and Predictions for ITER, Nuclear Fusion 56(2).

[4] Reux C., Lehnenb M., et al. 2013, Use of the disruption mitigation valve in closed loop for routine protection at JET, Fusion Engineering and Design 88 (2013) 1101- 1104.

[5] Maraschek M., et al. 2013, Measurement and impact of the $\mathrm{n}=1$ intrinsic error field at ASDEX Upgrade, 40 ${ }^{\text {th }}$ EPS Conference on Plasma Physics Espoo, Finland, 1st—5th July 2013.

[6] Zedda K. et al. 2007, An Example of a New Approach for the Development of Disruption Protection Tools for JET: The Mode-Lock Disruption Class, 34 ${ }^{\text {th }}$ EPS Conference on Plasma Physics, Warsaw, Poland 2nd - 6th July 2007.

[7] Vega J., Moreno R., Pereira A. et al. 2015, Advanced disruption predictor based on the locked mode signal: application to JET, Proceedings of Science, Vol. 2015-January, 2015, 1st EPS Conference on Plasma Diagnostics, ECPD 2015; Frascati; Italy; 14 - 17 April 2015.

[8] https://users.euro-fusion.org/pages/mags/equilibrium/eq-coil-loop/saddle-loop/saddleloop.htm

[9] Cannas B., Fanni A., Murari A., Pau A., Sias G., and JET EFDA Contributors 2013, Automatic Disruption Classification based on Manifold Learning for Real Time Applications on JET, Nucl. Fusion 53093023.

[10] Pau A., Cannas B., Fanni A., Sias G., Baruzzo M., Murari A., Pautasso G., Tsalas M., JET Contributors, the ASDEX Upgrade Team and the EUROfusion MST1 team 2017, A tool to support the construction of reliable disruption databases, Fusion Engineering and Design (2017), http://dx.doi.org/10.1016/j.fusengdes.2017.10.003. 
[11] Zhang Y., Pautasso G., et al. 2011, Prediction of disruptions on ASDEX Upgrade using discriminant analysis, Nuclear Fusion 51 (6).

[12] R. Aledda, B. Cannas, A. Fanni, A. Pau, G. Sias 2015, Improvements in disruption prediction at ASDEX Upgrade, Fusion Engineering and Design 96-97, 698-702. 